\title{
PLURIPOTENTIAL THEORY ON TEICHMÜLLER SPACE I: PLURICOMPLEX GREEN FUNCTION
}

\begin{abstract}
HIDEKI MIYACHI
ABstract. This is the first paper in a series of investigations of the pluripotential theory on Teichmüller space. One of the main purposes of this paper is to give an alternative approach to the Krushkal formula of the pluricomplex Green function on Teichmüller space. We also show that Teichmüller space carries a natural stratified structure of real-analytic submanifolds defined from the structure of singularities of the initial differentials of the Teichmüller mappings from a given point. We will also give a description of the Levi form of the pluricomplex Green function using the Thurston symplectic form via Dumas' symplectic structure on the space of holomorphic quadratic differentials.
\end{abstract}

\section{INTRODUCTION}

This is the first paper in a series of investigations of the pluripotential theory on Teichmüller space. One of the purposes of this paper is to give an alternative approach to a characterization of the pluricomplex Green function on Teichmüller space, which was first discussed by Krushkal in [36].

Theorem 1 (Pluricomplex Green function on Teichmüller space). Let $\mathcal{T}_{g, m}$ be the Teichmüller space of Riemann surfaces of analytically finite-type $(g, m)$, and let $d_{T}$ be the Teichmüller distance on $\mathcal{T}_{g, m}$. Then, the pluricomplex Green function $g_{\mathcal{T}_{g, m}}$ on $\mathcal{T}_{g, m}$ satisfies

$$
g_{\mathcal{T}_{g, m}}(x, y)=\log \tanh d_{T}(x, y)
$$

for $x, y \in \mathcal{T}_{g, m}$.

See $\$ 7.1$ for the definition of the pluricomplex Green function. In the second paper [4], we will establish the Poisson integral formula for pluriharmonic functions on Teichmüller space which are continuous on the Bers compactification. The Krushkal formula (1) of the pluricomplex Green function plays a crucial rule in the second paper. This result is announced in [47].

1.1. Results. From Klimek's work [29], it suffices for proving (11) to show that the right-hand side of (11) is plurisubharmonic (cf. 77.1). To show this, Krushkal applied Poletskii's characterization of the pluricomplex Green function (cf. [51]).

Received by the editors January 14, 2019.

2010 Mathematics Subject Classification. Primary 30F60, 32G15, 57M50, 31B05, 32U05, $32 \mathrm{U} 35$.

Key words and phrases. Singular Euclidean structures, Teichmüller space, Teichmüller distance, Levi forms, pluricomplex Green functions.

This work was partially supported by JSPS KAKENHI Grant Numbers 16K05202, 16H03933, $17 \mathrm{H} 02843$. 
Our strategy is a more direct method while looking ahead to future research (see $\$ 1.2$ ). Indeed, we calculate the Levi form of the log-tanh of the Teichmüller distance at generic points, and we check the non-negative definiteness ( $(7.6)$. The Levi form is a fundamental and standard invariant of plurisubharmonic functions in pluripotential theory (cf. [30]). From our calculation, we observe that the Levi form of the pluricomplex Green function is described by the Thurston symplectic form on the space of measured foliations via Dumas' symplectic structure on the space of holomorphic quadratic differentials (cf. [14]). This description implies a condition for deformations of Teichmüller mappings from a fixed base point to complex analytically varying targets from the topological aspect in Teichmüller theory (cf. 88 ). The calculation is established with the variations of the periods of holomorphic one forms on the double covering surfaces defined from initial and terminal Teichmüller differentials (cf. \$7).

1.1.1. The Demailly distance on $\mathcal{T}_{g, m}$. Let $\Omega$ be a hyperconvex domain in $\mathbb{C}^{N}$. Let $g_{\Omega}$ be the pluricomplex Green function on $\Omega$. Demailly [10, Théorème 5.3] defined a distance $\boldsymbol{\delta}_{\Omega}$ related to the pluricomplex Green function by

$$
\boldsymbol{\delta}_{\Omega}(z, w)=\limsup _{\zeta \rightarrow \partial \Omega}\left|\log \frac{g_{\Omega}(z, \zeta)}{g_{\Omega}(w, \zeta)}\right|, \quad(z, w \in \Omega) .
$$

The Demailly distance gives a Harnack-type inequality for the pluriharmonic Poisson kernel. To discuss the Demailly distance on $\mathcal{T}_{g, m}$, we identify $\mathcal{T}_{g, m}$ with the Bers slice with base point $x_{0} \in \mathcal{T}_{g, m}$ via the Bers embedding (cf. [2]). From Theorem 1, we observe the following pluripotential theoretic characterization of the Teichmüller distance, which will be proved in $\$ 7.7$

Corollary 1.1 (Demailly distance on $\mathcal{T}_{g, m}$ ). The Demailly distance on $\mathcal{T}_{g, m}$ coincides with twice of the Teichmüller distance.

1.1.2. Stratification of Teichmüller space and removable singularities. Dumas [14] gave a complex analytic stratification in the space $\mathcal{Q}_{x_{0}}$ of (non-zero) holomorphic quadratic differentials on $x_{0} \in \mathcal{T}_{g, m}$ in terms of the structure of singularities (cf. 6.1). Sending the stratification on $\mathcal{Q}_{x_{0}}$ by the Teichmüller homeomorphism (\$2.2.2), we obtain a topological stratification on $\mathcal{T}_{g, m}-\left\{x_{0}\right\}$. The top stratum $\mathcal{T}_{\infty}$ consists of $x \in \mathcal{T}_{g, m}-\left\{x_{0}\right\}$ such that the initial differential of the Teichmüller mapping from $x_{0}$ to $x$ is generic. We will show that the induced stratification on $\mathcal{T}_{g, m}-\left\{x_{0}\right\}$ is a real-analytic stratification in the sense that each stratum is a realanalytic submanifold (cf. Theorem 3). Applying the stratification, we shall show the following, which is crucial in our proof of Theorem 1 (cf. 86.3).

Theorem 2 (Non-generic strata are removable). A function of class $C^{1}$ on $\mathcal{T}_{g, m}-$ $\left\{x_{0}\right\}$ is plurisubharmonic on $\mathcal{T}_{g, m}$ if it is plurisubharmonic on the top stratum $\mathcal{T}_{\infty}$ and bounded above around $x_{0}$.

1.2. Backgrounds, motivation, and future. Teichmüller space $\mathcal{T}_{g, m}$ is a complex manifold which is homeomorphic to the Euclidean space. The infinitesimal complex structure is well-understood from the Kodaira-Spencer theory and the Ahlfors-Bers theory (cf. 26] and 49]). Regarding the global complex analytic property, it is known that Teichmüller space is realized as a polynomially convex and hyperconvex domain in the complex Euclidean space (cf. [26. Theorem 6.6], [55], and [35]), and the Teichmüller distance coincides with the Kobayashi distance under the complex structure (cf. [53]). 
On the other hand, to the author's knowledge, the global complex analytical structure is still less developed to discuss the end (boundary) of the Teichmüller space from the complex analytical viewpoint. In fact, it is conjectured that the Bers boundary of Teichmüller space is a fractal set in some sense (cf. [7, Question 10.7 in $§ 10.3]$ ). To analyse the geometry of the Bers boundary, we need to understand the behavior of holomorphic functions (holomorphic local coordinates) around the Bers boundary which are defined on the Bers closure, such as the trace functions derived from projective structures or Kleinian groups. Actually, the complex length functions, which are defined from the trace functions, are roughly estimated with topological invariants around the Bers boundary in the proof of the ending lamination theorem (cf. [42, [43], and [6]), and the estimations turn out to be very important and useful estimates for studying the boundary (e.g., 33 and 44]). However, it seems to be expected we will need sharper estimates with topological invariants for investigating the geometry of the Bers boundary to establish the conjecture.

According to Thurston theory, the end of the Teichmüller space from the topological viewpoint consists of the degenerations of complex or hyperbolic structures on a reference surface via the geometric intersection numbers (cf. [12]). The Teichmüller distance and the extremal length have appeared in quasiconformal geometry on Riemann surfaces. Recently, they have been thought of as geometric intersection numbers under the Gardiner-Masur compactification, and we give a connection between quasiconformal geometry and Thurston theory (extremal length geometry) (cf. [18, [28], and [45]).

Pluripotential theory is a powerful theory for investigating complex manifolds. The pluricomplex Green function is one of many important functions in pluripotential theory. The pluricomplex Green function is a fundamental solution of the Dirichlet problem relative to the Monge-Ampère operator, and defines the pluriharmonic measures on the boundaries for hyperconvex domains (cf. [10] and 29]; see also 97.1). The asymptotic behavior of the pluricomplex Green function on a domain is sensitive in terms of the regularity of the boundary (cf. [9], 11], 22]).

Since Teichmüller space is hyperconvex, from Demailly's theory [10, $\mathcal{T}_{g, m}$ admits a unique pluricomplex Green function. By virtue of the ending lamination theorem, the Bers boundary is parametrized by the topological invariants called the ending invariants. By Demailly's Poisson integral formula formulated with the pluriharmonic measures ([10]), holomorphic functions around the Bers closure are represented with their boundary functions which are defined on a space with topological background, and are studied from a bird's-eye view in complex function theory.

As a conclusion, the Krushkal formula (1) and further investigations on the pluricomplex Green function are expected to strengthen mutual interaction among quasiconformal geometry, the complex analytic (pluripotential theoretic) aspect, and the topological aspect (Thurston theory) in Teichmüller theory.

1.3. About the paper. This paper is organized as follows. From to 4 we recall the basic notion and properties in Teichmüller theory. In $\$ 5$ we discuss the deformation of singular Euclidean structures associated to the Teichmüller deformations from a fixed point $x_{0} \in \mathcal{T}_{g, m}$. In $\$ 6$, we will give the stratification on $\mathcal{T}_{g, m}-\left\{x_{0}\right\}$. We show Theorem 1 and Corollary 1.1 in 97 and discuss the topological description of the Levi form in 8 . 


\section{TEICHMÜLlER THEORY}

Let $\Sigma_{g, m}$ be a closed orientable surface of genus $g$ with $m$-marked points with $2 g-2+m>0$ (possibly $m=0$ ). In this section, we recall basics in Teichmüller theory. For reference, see [12], [17], 25], 26], and 49] for instance.

2.1. Teichmüller space. Teichmüller space $\mathcal{T}_{g, m}$ is the set of equivalence classes of marked Riemann surfaces of type $(g, m)$. A marked Riemann surface $(M, f)$ of type $(g, m)$ is a pair of a Riemann surface $M$ of analytically finite-type $(g, m)$ and an orientation preserving homeomorphism $f: \Sigma_{g, m} \rightarrow M$. Two marked Riemann surfaces $\left(M_{1}, f_{1}\right)$ and $\left(M_{2}, f_{2}\right)$ of type $(g, m)$ are (Teichmüller) equivalent if there is a conformal mapping $h: M_{1} \rightarrow M_{2}$ such that $h \circ f_{1}$ is homotopic to $f_{2}$.

The Teichmüller distance $d_{T}$ is a complete distance on $\mathcal{T}_{g, m}$ defined by

$$
d_{T}\left(x_{1}, x_{2}\right)=\frac{1}{2} \log \inf _{h} K(h)
$$

for $x_{i}=\left(M_{i}, f_{i}\right)(i=1,2)$, where the infimum runs over all quasiconformal mapping $h: M_{1} \rightarrow M_{2}$ homotopic to $f_{2} \circ f_{1}^{-1}$, and $K(h)$ is the maximal dilatation of a quasiconformal mapping $h$.

2.2. Quadratic differentials and infinitesimal complex structure on $\mathcal{T}_{g, m}$. For $x=(M, f) \in \mathcal{T}_{g, m}$, we denote by $\mathcal{Q}_{x}$ the complex Banach space of holomorphic quadratic differentials $q=q(z) d z^{2}$ on $M$ with $L^{1}$-norm

$$
\|q\|=\int_{M}|q(z)| \frac{\sqrt{-1}}{2} d z \wedge d \bar{z}<\infty .
$$

From the Riemann-Roch theorem, the space $\mathcal{Q}_{x}$ is isomorphic to $\mathbb{C}^{3 g-3+m}$. Let

$$
\Pi: \mathcal{Q}_{g, m}=\cup_{x \in \mathcal{T}_{g, m}} \mathcal{Q}_{x} \rightarrow \mathcal{T}_{g, m}
$$

be the complex vector bundle of quadratic differentials over $\mathcal{T}_{g, m}$. A differential $q \in \mathcal{Q}_{g, m}$ is said to be generic if all zeros are simple and all marked points of the underlying surface are simple poles of $q$. Generic differentials are open and dense subsets in $\mathcal{Q}_{g, m}$ and in each fiber $\mathcal{Q}_{x}$ for $x \in \mathcal{T}_{g, m}$.

2.2.1. Infinitesimal complex structure. Teichmüller space $\mathcal{T}_{g, m}$ is a complex manifold of dimension $3 g-3+m$. The infinitesimal complex structure is described as follows: Let $x=(M, f) \in \mathcal{T}_{g, m}$. Let $L^{\infty}(M)$ be the Banach space of measurable $(-1,1)$-forms $\mu=\mu(z) d \bar{z} / d z$ on $M$ with

$$
\|\mu\|_{\infty}=\operatorname{ess.} \sup _{p \in M}|\mu(p)|<\infty .
$$

The holomorphic tangent space $T_{x} \mathcal{T}_{g, m}$ at $x$ of $\mathcal{T}_{g, m}$ is described as the quotient space

where

$$
L^{\infty}(M) /\left\{\mu \in L^{\infty}(M) \mid\langle\mu, \varphi\rangle=0 \forall \varphi \in \mathcal{Q}_{x}\right\}
$$

$$
\langle\mu, \varphi\rangle=\int_{M} \mu(z) \varphi(z) \frac{\sqrt{-1}}{2} d z \wedge d \bar{z} .
$$

For $v=[\mu] \in T_{x} \mathcal{T}_{g, m}$ and $\varphi \in \mathcal{Q}_{x}$, the canonical pairing between $T_{x} \mathcal{T}_{g, m}$ and $\mathcal{Q}_{x}$ is defined by

$$
\langle v, \varphi\rangle=\langle\mu, \varphi\rangle
$$

and it induces an identification between $\mathcal{Q}_{x}$ and the holomorphic cotangent space $T_{x}^{*} \mathcal{T}_{g, m}$. 
2.2.2. The Teichmüller homeomorphism. Let $\mathcal{U} \mathcal{Q}_{x}$ be the unit ball in $\mathcal{Q}_{x}$. For $q \in$ $\mathcal{U} \mathcal{Q}_{x}$, we define a quasiconformal mapping $f^{q}$ on $M$ from the Beltrami differential $\|q\|(\bar{q} /|q|) \in L^{\infty}(M)$. Then, $\mathcal{T}_{g, m}$ is homeomorphic to $\mathcal{U} \mathcal{Q}_{x}$ with

$$
\Xi=\Xi_{x}: \mathcal{U} \mathcal{Q}_{x} \ni q \mapsto\left(f^{q}(M), f^{q} \circ f\right) \in \mathcal{T}_{g, m} .
$$

We call the homeomorphism (4) the Teichmüller homeomorphism. The Teichmüller homeomorphism gives a useful representation of the Teichmüller distance as

$$
d_{T}\left(x, \Xi_{x}(q)\right)=\frac{1}{2} \log \frac{1+\|q\|}{1-\|q\|}=\tanh ^{-1}(\|q\|)
$$

for $q \in \mathcal{U} \mathcal{Q}_{x}$,

2.3. Measured foliations. Let $\mathcal{S}$ be the set of homotopy classes of non-trivial and non-peripheral simple closed curves on $\Sigma_{g, m}$. Let $i(\alpha, \beta)$ denote the geometric intersection number for simple closed curves $\alpha, \beta \in \mathcal{S}$. Let $\mathcal{W S}=\{t \alpha \mid t \geq 0, \alpha \in$ $\mathcal{S}\}$ be the set of weighted simple closed curves. The set $\mathcal{S}$ is canonically identified with a subset of $\mathcal{W S}$ as weight 1 curves.

We consider an embedding

$$
\mathcal{W S} \ni t \alpha \mapsto[\mathcal{S} \ni \beta \mapsto t i(\alpha, \beta)] \in \mathbb{R}_{\geq 0}^{\mathcal{S}} .
$$

We topologize the function space $\mathbb{R}_{\geq 0}^{\mathcal{S}}$ with the topology of pointwise convergence. The closure $\mathcal{M F}$ of the image of the embedding is called the space of measured foliations on $\Sigma_{g, m}$. The space $\mathcal{M F}$ is homeomorphic to $\mathbb{R}^{6 g-6+2 m}$, and contains the weighted simple closed curves $\mathcal{W S}$ as a dense subset. The intersection number on $\mathcal{W S}$ is defined by $i(t \alpha, s \beta)=t s i(\alpha, \beta)$ for $t \alpha, s \beta \in \mathcal{W S}$. The intersection number extends continuously as a non-negative function $i(\cdot, \cdot)$ on $\mathcal{M F} \times \mathcal{M F}$ with $i(F, F)=0$ and $F(\alpha)=i(F, \alpha)$ for $F \in \mathcal{M F} \subset \mathbb{R}_{\geq 0}^{\mathcal{S}}$ and $\alpha \in \mathcal{S}$.

2.4. Hubbard-Masur differentials and extremal length. Let $x=(M, f) \in$ $\mathcal{T}_{g, m}$. For $q \in \mathcal{Q}_{x}$, the vertical foliation $v(q)$ of $q$ is a measured foliation defined by

$$
i(v(q), \alpha)=\inf _{\alpha^{\prime} \in f(\alpha)} \int_{\alpha^{\prime}}|\operatorname{Re}(\sqrt{q})|
$$

for $\alpha \in \mathcal{S}$. Hubbard and Masur observed that the correspondence, which we call the Hubbard-Masur homeomorphism,

$$
\mathcal{V}_{x}: \mathcal{Q}_{x} \ni q \mapsto v(q) \in \mathcal{M F},
$$

is a homeomorphism (cf. [24] and Remark 8.1). For $F \in \mathcal{M F}$, the HubbardMasur differential $q_{F, x}$ for $F$ at $x$ is defined to satisfy $v\left(q_{F, x}\right)=F$. By definition, $q_{t F, x}=t^{2} q_{F, x}$ for $F \in \mathcal{M F}$ and $t \geq 0$.

For $F \in \mathcal{M F}$, the extremal length of $F$ at $x$ is defined by

$$
\operatorname{Ext}_{x}(F)=\left\|q_{F, x}\right\| .
$$

Kerckhoff [28] observed that the Teichmüller distance is expressed as

$$
d_{T}(x, y)=\frac{1}{2} \log \sup _{\alpha \in \mathcal{S}} \frac{\operatorname{Ext}_{x}(\alpha)}{\operatorname{Ext}_{y}(\alpha)}
$$

for $x, y \in \mathcal{T}_{g, m}$. This expression is called the Kerckhoff formula of the Teichmüller distance. 


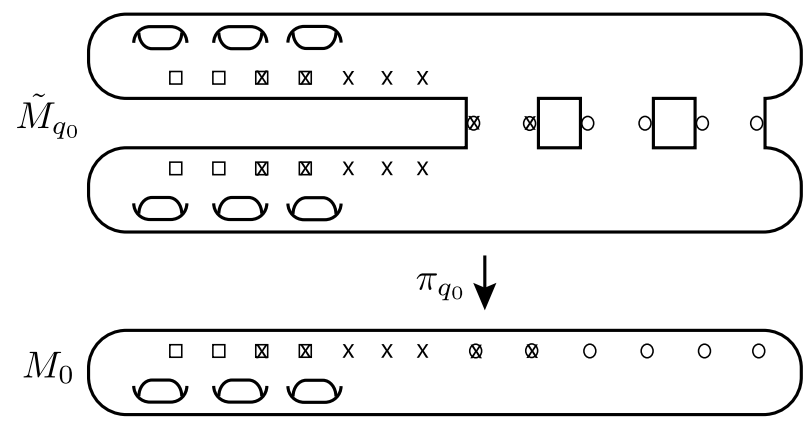

Figure 1. The covering space $\pi_{q_{0}}: \tilde{M}_{q_{0}} \rightarrow M_{0}$ : Symbols $\circ, \square$, and $x$ in the figure mean non-orientable singularities, orientable singularities, and marked points, respectively. Each singularity may coincide with a marked point (denoted by $\otimes$ for non-orientable singularities and by $\otimes$ otherwise). Points $\otimes$ may or may not be poles of $q_{0}$. In our notation, $\Sigma\left(q_{0}\right)=\{\circ, \otimes, \square, \otimes, \times\}, \Sigma_{s}\left(q_{0}\right)=$ $\{\circ, \otimes, \square, \otimes\}, \Sigma_{s m}\left(q_{0}\right)=\{\otimes, \otimes\}, \Sigma_{m \backslash s}\left(q_{0}\right)=\{\times\}, \Sigma_{s \backslash m}\left(q_{0}\right)=$ $\{\circ, \square\}, \Sigma_{u b}\left(q_{0}\right)=\{\times, \square, \otimes\}, \Sigma_{o}\left(q_{0}\right)=\{\circ, \otimes\}$, and $\Sigma_{e}\left(q_{0}\right)=$ $\{\square, \otimes\}$.

\section{Double COVERING SPACES ASSOCIATED TO QUADRATIC DIFFERENTIALS}

3.1. Branched covering spaces. Let $x_{0}=\left(M_{0}, f_{0}\right) \in \mathcal{T}_{g, m}$. Let $\Sigma_{m}=\Sigma_{m}\left(x_{0}\right)$ be the marked points of $M_{0}$. Let $q_{0} \in \mathcal{Q}_{x_{0}} \subset \mathcal{Q}_{g, m}$. Let $\Sigma_{s}\left(q_{0}\right)$ be the set of singularities of $q_{0}$. In accordance with [1], a singular point of $q_{0}$ is called orientable if it is of even order, and non-orientable otherwise. Any orientable singular point is a zero of $q_{0}$. Let $\Sigma_{o}=\Sigma_{o}\left(q_{0}\right)$ (resp., $\Sigma_{e}=\Sigma_{e}\left(q_{0}\right)$ ) be the set of non-orientable (resp., orientable) zeros of $q_{0}$ (" $o$ " and " $e$ " stand for "odd" and "even"). The number of non-orientable singularities is always even. By definition, $\Sigma_{s}\left(q_{0}\right)=\Sigma_{o}\left(q_{0}\right) \cup \Sigma_{e}\left(q_{0}\right)$. We define

$$
\begin{aligned}
\Sigma\left(q_{0}\right) & =\Sigma_{e}\left(q_{0}\right) \cup \Sigma_{o}\left(q_{0}\right) \cup \Sigma_{m}\left(x_{0}\right), \\
\Sigma_{s m}\left(q_{0}\right) & =\Sigma_{s}\left(q_{0}\right) \cap \Sigma_{m}\left(x_{0}\right), \\
\Sigma_{s \backslash m}\left(q_{0}\right) & =\Sigma_{s}\left(q_{0}\right) \backslash \Sigma_{m}\left(x_{0}\right), \\
\Sigma_{m \backslash s}\left(q_{0}\right) & =\Sigma_{m}\left(x_{0}\right) \backslash \Sigma_{s}\left(q_{0}\right), \\
\Sigma_{u b}\left(q_{0}\right) & =\Sigma_{e}\left(q_{0}\right) \sqcup \Sigma_{m \backslash s}\left(q_{0}\right) .
\end{aligned}
$$

We call a marked point in $\Sigma_{m \backslash s}\left(q_{0}\right)$ free. The set $\Sigma\left(q_{0}\right)$ is the totality of marked points caused by $q_{0}$, and it is represented as the disjoint unions

$$
\Sigma\left(q_{0}\right)=\Sigma_{u b}\left(q_{0}\right) \sqcup \Sigma_{o}\left(q_{0}\right) .
$$

Consider the double branched covering space $\pi_{q_{0}}: \tilde{M}_{q_{0}} \rightarrow M_{0}$ of the square root $\sqrt{q_{0}}$ (cf. Figure 1). For $p \in \Sigma\left(q_{0}\right)$, the preimage $\pi_{q_{0}}^{-1}(p)$ consists of two points if and only if $p \in \Sigma_{u b}\left(q_{0}\right)$ ("ub" stands for "unbranched"). The projection $\pi_{q_{0}}$ is 
double-branched over points in $\tilde{\Sigma}_{o}\left(q_{0}\right)$. The surface $\tilde{M}_{q_{0}}$ is a closed surface of genus

$$
\tilde{g}\left(q_{0}\right)= \begin{cases}2 g-1+\frac{1}{2} \#_{\Sigma_{o}}\left(q_{0}\right) & \left(q_{0}\right. \text { is not square) } \\ 2 g & \text { (otherwise) }\end{cases}
$$

(cf. [24, §2]). In particular, the surface $\tilde{M}_{q_{0}}$ is a closed Riemann surface of genus $4 g-3+m$ when $q_{0}$ is generic. The square root $\sqrt{q_{0}}$ on $M_{0}$ is lifted as the abelian differential $\omega_{q_{0}}$ on $\tilde{M}_{q_{0}}$. The covering transformation $i_{q_{0}}$ of the covering is a conformal involution on $\tilde{M}_{q_{0}}$ which satisfies $\pi_{q_{0}} \circ i_{q_{0}}=\pi_{q_{0}}$ and $i_{q_{0}}^{*} \omega_{q_{0}}=-\omega_{q_{0}}$. For each set $\Sigma_{\bullet}\left(q_{0}\right)$ defined above, we denote by $\tilde{\Sigma}_{\bullet}\left(q_{0}\right)$ the preimage of $\Sigma_{\bullet}\left(q_{0}\right)$. When $q_{0}$ is square in the sense that $q_{0}=\omega^{2}$ for some abelian differential $\omega$ on $M_{0}, \tilde{M}_{q_{0}}$ consists of two copies of $M_{0}$. We consider the pair $\left(\tilde{M}_{q_{0}}, \tilde{\Sigma}_{u b}\left(q_{0}\right)\right)$ as a Riemann surface with marked points.

Convention. Let $V$ be a vector space $V$ with an involution. We denote by $V^{ \pm}$ the eigenspace in $V$ of the eigenvalue \pm 1 of the action of the involution.

We also recall the following elementary fact: For vector spaces $V_{i}$ with an involution $(i=1,2,3)$, an exact sequence $0 \rightarrow V_{1} \rightarrow V_{2} \rightarrow V_{3} \rightarrow 0$ commuting the involutions induces an exact sequence $0 \rightarrow V_{1}^{-} \rightarrow V_{2}^{-} \rightarrow V_{3}^{-} \rightarrow 0$.

3.2. A subspace in quadratic differentials. For $q_{0} \in \mathcal{Q}_{x_{0}}$ and $x_{0}=\left(M_{0}, f_{0}\right)$, we define

$$
\mathcal{Q}_{x_{0}}^{T}\left(q_{0}\right)=\left\{\psi \in \mathcal{Q}_{x_{0}} \mid(\psi) \geq \prod_{p \in \Sigma_{s \backslash m}\left(q_{0}\right)} p^{\boldsymbol{o}_{p}\left(q_{0}\right)-1} \prod_{p \in \Sigma_{s m}\left(q_{0}\right)} p^{\boldsymbol{o}_{p}\left(q_{0}\right)}\right\},
$$

where $\boldsymbol{o}_{p}\left(q_{0}\right)$ is the order of $q_{0}$ at $p \in M_{0}$, and $(\psi)$ is the divisor of $\psi$. The symbol "T" stands for "tangent". When $M_{0}$ has no marked point (i.e., $m=0$ ), $\psi \in \mathcal{Q}_{x_{0}}^{T}\left(q_{0}\right)$ is equivalent to the condition that $\psi / q_{0}$ has at most simple poles on $M_{0}$ (cf. [14, Lemma 5.2]; see also Proposition 6.1 below). Notice that $\mathcal{Q}_{x_{0}}^{T}\left(q_{0}\right)=\mathcal{Q}_{x_{0}}$ if $q_{0}$ is generic.

3.3. The $q$-realizations of tangent vectors. Let $x_{0}=\left(M_{0}, f_{0}\right) \in \mathcal{T}_{g, m}$ and $q_{0} \in \mathcal{Q}_{x_{0}}-\{0\}$ be a generic differential. For $v \in T_{x_{0}} \mathcal{T}_{g, m}$, a holomorphic quadratic differential $\eta_{v} \in \mathcal{Q}_{x_{0}}$ is said to be the $q_{0}$-realization of $v$ if it satisfies

$$
\langle v, \psi\rangle_{x_{0}}=\int_{M_{0}} \frac{\overline{\eta_{v}}}{\left|q_{0}\right|} \psi
$$

for all $\psi \in \mathcal{Q}_{x_{0}}$ where $x_{0}=\left(M_{0}, f_{0}\right) \in \mathcal{T}_{g, m}$ (cf. [46]). Notice that $\eta_{v}$ in (10) does exist because

$$
\left(\psi_{1}, \psi_{2}\right) \mapsto \int_{M_{0}} \frac{\psi_{1} \overline{\psi_{2}}}{\left|q_{0}\right|}
$$

is a non-degenerate Hermitian inner product on $\mathcal{Q}_{x_{0}}$ (cf. [14, §5]). The correspondence

$$
T_{x_{0}} \mathcal{T}_{g, m} \ni v \mapsto \eta_{v} \in \mathcal{Q}_{x_{0}}
$$


is an anti-complex linear isomorphism. The Hermitian form (11) is calculated as

$$
\begin{aligned}
\int_{M_{0}} \frac{\psi_{1} \overline{\psi_{2}}}{\left|q_{0}\right|}= & \frac{\sqrt{-1}}{4} \int_{\tilde{M}_{q_{0}}} \frac{\pi_{q_{0}}^{*}\left(\psi_{1}\right)}{\omega_{q_{0}}} \wedge \overline{\left(\frac{\pi_{q_{0}}^{*}\left(\psi_{2}\right)}{\omega_{q_{0}}}\right)} \\
= & \frac{1}{2} \int_{\tilde{M}_{q_{0}}} \operatorname{Re}\left(\frac{\pi_{q_{0}}^{*}\left(\psi_{1}\right)}{\omega_{q_{0}}}\right) \wedge \operatorname{Im}\left(\frac{\pi_{q_{0}}^{*}\left(\psi_{2}\right)}{\omega_{q_{0}}}\right) \\
& +\frac{\sqrt{-1}}{2} \int_{\tilde{M}_{q_{0}}} \operatorname{Re}\left(\frac{\pi_{q_{0}}^{*}\left(\psi_{1}\right)}{\omega_{q_{0}}}\right) \wedge \operatorname{Re}\left(\frac{\pi_{q_{0}}^{*}\left(\psi_{2}\right)}{\omega_{q_{0}}}\right)
\end{aligned}
$$

for $\psi_{1}, \psi_{2} \in \mathcal{Q}_{x_{0}}$.

\section{Stratifications ON $\mathcal{Q}_{g, m}$}

4.1. Stratification. Following Dumas [14, we recall the definition of stratifications on manifolds. Let $Z$ be a manifold. A stratification of $Z$ is a locally finite collection of locally closed submanifolds $\left\{Z_{i}\right\}_{i \in I}$ of $Z$, the strata, indexed by a set $I$ such that

(1) $Z=\bigcup_{j \in I} Z_{j}$

(2) $Z_{j} \cap \overline{Z_{k}} \neq \emptyset$ if and only if $Z_{j} \subset \overline{Z_{k}}$.

From the second condition, $Z_{i} \cap Z_{j} \neq \emptyset$ if and only if $Z_{i}=Z_{j}$ because each $Z_{i}$ is locally closed. A stratification of a complex manifold $Z$ is a complex analytic stratification if the closure $\overline{Z_{j}}$ and the boundary $\overline{Z_{j}} \backslash Z_{j}$ of each stratum $Z_{j}$ are complex analytic sets.

4.2. Strata in $\mathcal{Q}_{g, m}$. Our strata and symbol are slightly different from that treated by Masur-Smillie [41] and Veech [59. We consider here the deformation of quadratic differentials with marked points for our purpose (see also 4.3 below). If any marked point of a given quadratic differential is a singular point, our strata coincides with their strata (cf. [59, §1]).

A symbol of $q \in \mathcal{Q}_{g, m}-\{0\}$ is a quadruple $\boldsymbol{\pi}=(\boldsymbol{m}, \boldsymbol{n}(-1), \boldsymbol{n}(\cdot), \varepsilon)$ where $\boldsymbol{m}$ is the number of free marked points, $\boldsymbol{n}(-1)$ is the number of poles, $\boldsymbol{n}(l)$ is the number of zeros of order $l \geq 1$, and $\varepsilon= \pm 1$ according to whether $q$ is square $(\varepsilon=1)$ or not $(\varepsilon=-1)$. We set $\boldsymbol{n}(0)=0$ for simplicity. Notice that $\sum_{l \geq-1} l \cdot \boldsymbol{n}(l)=4 g-4$. Let $\mathcal{Q}(\boldsymbol{\pi})=\mathcal{Q}_{g, m}(\boldsymbol{\pi}) \subset \mathcal{Q}_{g, m}$ be the set of holomorphic quadratic differentials in $\mathcal{Q}_{g, m}$ whose symbol is $\boldsymbol{\pi}$. As we discuss in Proposition 4.1 below, each component of $\mathcal{Q}(\boldsymbol{\pi})$ is a complex manifold of dimension

$$
\operatorname{dim}_{\mathbb{C}} \mathcal{Q}(\boldsymbol{\pi})=2 g+\frac{\varepsilon-3}{2}+\boldsymbol{m}+\sum_{l \geq-1} \boldsymbol{n}(l) .
$$

Let $\boldsymbol{\pi}(q)=\left(\boldsymbol{m}_{q}, \boldsymbol{n}_{q}(-1), \boldsymbol{n}_{q}(\cdot), \varepsilon_{q}\right)$ be the symbol of $q \in \mathcal{Q}_{g, m}$. If $\Sigma_{s}(q)=\emptyset$, we have $g=1$ and $q$ is square. We set $\boldsymbol{\pi}(q)=(m, 0,\{0, \cdots\}, 1)$ in this case.

Since $\boldsymbol{m}_{q}+\sum_{l \geq-1} \boldsymbol{n}_{q}(l)={ }^{\#} \Sigma(q)={ }^{\#} \Sigma_{0}(q)+{ }^{\#} \Sigma_{u b}(q)$ for $q \in \mathcal{Q}_{g, m}$, from (13), we can check the following:

$$
\operatorname{dim}_{\mathbb{C}} \mathcal{Q}(\boldsymbol{\pi}(q))=\operatorname{dim}_{\mathbb{C}} \operatorname{Hom}\left(H_{1}\left(\tilde{M}_{q}, \tilde{\Sigma}_{u b}(q), \mathbb{R}\right)^{-}, \mathbb{C}\right) .
$$


4.3. Remark on the stratification on $\mathcal{Q}_{g, m}$. Our stratification of $\mathcal{Q}_{g, m}$ is slightly different from Masur-Smillie-Veech's one in the following sense: We have mainly two differences from their stratification:

(1) If a free marked point and a singular point collide in a moving of quadratic differentials, we recognize the quadratic differentials to be degenerating into the other stratum. Two free marked points cannot collide because we consider the deformation on $\mathcal{T}_{g, m}$; and

(2) if a singular point of a quadratic differential in a stratum lies at a marked point, the singular point stays on the marked point in deforming on the stratum for the quadratic differential.

All of these phenomena can be handled by standard arguments with complex analysis (for instance, [41] and [58]).

4.4. Masur-Smillie-Veech charts of the strata in $\mathcal{Q}_{g, m}$. For $q_{0} \in \mathcal{Q}\left(\boldsymbol{\pi}\left(q_{0}\right)\right)$, the union $\bigcup_{q} \tilde{M}_{q}$ is regarded as a trivial bundle over a small contractible neighborhood of $q_{0}$ whose fiber is a (possibly disconnected) surface with marked points. For each $q \in \mathcal{Q}\left(\boldsymbol{\pi}\left(q_{0}\right)\right)$ which is sufficiently close to $q_{0}$, the surface $\tilde{M}_{q}$ admits a marking inherited from the product structure of the bundle. Hence, we can identify $H_{1}\left(\tilde{M}_{q}, \Sigma_{u b}(q), \mathbb{R}\right)^{-}$and $H_{1}\left(\tilde{M}_{q}, \Sigma_{u b}(q), \mathbb{R}\right)$ with $H_{1}\left(\tilde{M}_{q_{0}}, \Sigma_{u b}\left(q_{0}\right), \mathbb{R}\right)^{-}$and $H_{1}\left(\tilde{M}_{q_{0}}, \Sigma_{u b}\left(q_{0}\right), \mathbb{R}\right)$ for $q \in \mathcal{Q}\left(\boldsymbol{\pi}\left(q_{0}\right)\right)$ near $q_{0}$ in the canonical manner.

The following is well known (e.g., [41, 40], [58, and [59]).

Proposition 4.1 (Local chart). There is a neighborhood $V_{0}$ of $q_{0}$ in $\mathcal{Q}\left(\boldsymbol{\pi}\left(q_{0}\right)\right)$ such that the mapping

$$
\Phi_{0}: V_{0} \ni q \mapsto\left[C \mapsto \int_{C} \omega_{q}\right] \in \operatorname{Hom}\left(H_{1}\left(\tilde{M}_{q_{0}}, \Sigma_{u b}\left(q_{0}\right), \mathbb{R}\right)^{-}, \mathbb{C}\right)
$$

is a holomorphic local chart around $q_{0}$.

\section{Deformations of QUAdratiC Differentials}

Henceforth, we set $\operatorname{Hom}\left(q_{0}\right)=\operatorname{Hom}\left(H_{1}\left(\tilde{M}_{q_{0}}, \tilde{\Sigma}_{u b}\left(q_{0}\right), \mathbb{R}\right)^{-}, \mathbb{C}\right)$ for the simplicity. From Proposition 4.1 $T_{q_{0}} \mathcal{Q}\left(\boldsymbol{\pi}\left(q_{0}\right)\right)$ is isomorphic to $\operatorname{Hom}\left(q_{0}\right)$ as $\mathbb{C}$-vector spaces.

In this section, to describe the deformations via the periods geometrically, we consider a $\Delta$-complex structure on $\tilde{M}_{q_{0}}$ for given $q_{0} \in \mathcal{Q}_{g, m}$, and describe the infinitesimal deformations along elements in $T_{q_{0}} \mathcal{Q}\left(\boldsymbol{\pi}\left(q_{0}\right)\right) \cong \operatorname{Hom}\left(q_{0}\right)$ by piecewise affine deformations. The description was already discussed by various authors (e.g., [41] and [59]). We also discuss it for the completeness.

5.1. $\Delta$-complex structure. A $\Delta$-complex structure on a space $X$ is a collection of a singular simplex $\sigma_{\alpha}: \Delta^{n} \rightarrow X\left(\Delta^{n}\right.$ is the standard $n$-simplex), with $n=n(\alpha)$ such that

(1) the restriction $\sigma_{\alpha}$ to the interior of $\Delta^{n}$ is injective, and each point of $X$ is in the image of exactly one such restriction;

(2) each restriction of $\sigma_{\alpha}$ to a face of $\Delta^{n}$ is one of the maps $\sigma_{\beta}: \Delta^{n-1} \rightarrow X$. Here, we are identifying the face of $\Delta^{n}$ with $\Delta^{n-1}$ by the canonical linear homeomorphism between them that preserves the ordering of the vertices; and

(3) a set $A \subset X$ is open if and only if $\sigma_{\alpha}^{-1}(A)$ is open in $\Delta^{n}$ for each $\sigma_{\alpha}$ (cf. [21, $\S 2.1])$. 


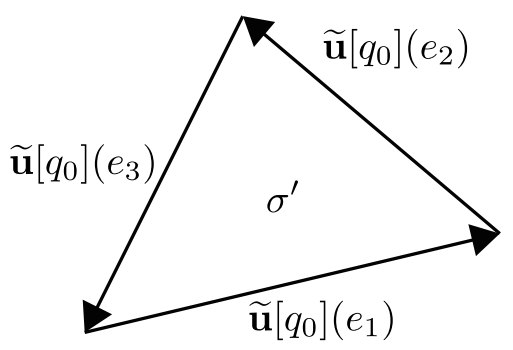

Figure 2. Euclidean triangle from $\omega_{q_{0}}$.

A $\Delta$-complex structure on a surface gives a kind of triangulation. The (relative) (co)homology group defined by a $\Delta$-complex structure on a space $X$ coincides with the (relative) (co)homology group of $X$ (cf. [21]).

5.2. Singular Euclidean structure on $\tilde{M}_{q_{0}}$. Let $x_{0}=\left(M_{0}, f_{0}\right) \in \mathcal{T}_{g, m}$ and $q_{0} \in \mathcal{Q}_{x_{0}}$. Consider a $\Delta$-complex structure $\Delta$ on $M_{0}$ such that the 0 -skeleton $\Delta^{(0)}$ contains $\Sigma\left(q_{0}\right)$, each 1-simplex is a straight segment with respect to the $\left|q_{0}\right|$-metric, and each 2 -simplex is a non-degenerate triangle. Such a $\Delta$-complex exists. For instance, we can take it as a refinement (subdivision) of the Delaunay triangulation with respect to the singularities of $q_{0}$ (cf. [41, §4]). Let $\tilde{\Delta}$ be the lift of $\Delta . \tilde{\Delta}$ is a $\Delta$-complex structure on $\tilde{M}_{q_{0}}$. The covering transformation $i_{q_{0}}$ acts on the 1-chain group $C_{1}\left(\tilde{\Delta}, \tilde{\Sigma}_{u b}\left(q_{0}\right), \mathbb{R}\right)$.

We define $\widetilde{\mathbf{u}}\left[q_{0}\right] \in \operatorname{Hom}\left(C_{1}\left(\tilde{\Delta}, \tilde{\Sigma}_{u b}\left(q_{0}\right)\right)^{-}, \mathbb{C}\right)$ by

$$
\widetilde{\mathbf{u}}\left[q_{0}\right](e)=\int_{e} \omega_{q_{0}}
$$

for $e \in C_{1}\left(\tilde{\Delta}, \Sigma_{u b}\left(q_{0}\right)\right)^{-}$. The restriction of $\widetilde{\mathbf{u}}\left[q_{0}\right]$ to the cycles $Z_{1}\left(\tilde{\Delta}, \Sigma_{u b}\left(q_{0}\right), \mathbb{R}\right)^{-}$ descends to a homomorphism $\mathbf{u}\left[q_{0}\right] \in \operatorname{Hom}\left(q_{0}\right)$ such that $\Phi_{0}\left(q_{0}\right)=\mathbf{u}\left[q_{0}\right]$ (cf. Proposition 4.1).

5.3. Piecewise affine deformations. Let $\sigma$ be a 2-simplex in $\Delta$. Let $\partial \sigma=$ $e_{1}+e_{2}+e_{3}$ as 1-chains. The developing mapping $\sigma \ni p \mapsto z(p)=\int^{p} \omega_{q_{0}}$ maps $\sigma$ to a Euclidean triangle $\sigma^{\prime}$ in the complex plane $\mathbb{C}$ with (oriented) edges $\widetilde{\mathbf{u}}\left[q_{0}\right]\left(e_{i}\right)$. Notice that $d z=\omega_{q_{0}}$ on $\sigma^{\prime}$ (cf. Figure 2).

For $\mathbf{v} \in \operatorname{Hom}\left(q_{0}\right) \cong T_{q_{0}} \mathcal{Q}\left(\boldsymbol{\pi}\left(q_{0}\right)\right)$, the infinitesimal deformation along $\mathbf{v}$ of the singular Euclidean structure associated to $q_{0}$ is described by an assortment of the affine deformation of the triangle $\sigma$ along the lift $\widetilde{\mathbf{v}} \in \operatorname{Hom}\left(C_{1}\left(\tilde{\Delta}, \tilde{\Sigma}_{u b}\left(q_{0}\right), \mathbb{R}\right)^{-}, \mathbb{C}\right)$ of $\mathbf{v}$. Here, we define the lift $\widetilde{\mathbf{v}}$ as follows: We first take the pullback of $\mathbf{v}$ on $Z_{1}\left(\tilde{\Delta}, \tilde{\Sigma}_{u b}\left(q_{0}\right), \mathbb{R}\right)^{-}$by precomposing the projection from $Z_{1}\left(\tilde{\Delta}, \tilde{\Sigma}_{u b}\left(q_{0}\right), \mathbb{R}\right)^{-}$to $H_{1}\left(\tilde{M}_{q_{0}}, \tilde{\Sigma}_{u b}\left(q_{0}\right), \mathbb{R}\right)^{-}$, and set $\widetilde{\mathbf{v}} \equiv 0$ on a complementary space of $Z_{1}\left(\tilde{\Delta}, \tilde{\Sigma}_{u b}\left(q_{0}\right), \mathbb{R}\right)^{-}$ in $C_{1}\left(\tilde{\Delta}, \tilde{\Sigma}_{u b}\left(q_{0}\right), \mathbb{R}\right)^{-}$.

To be more precise, fix a norm on $\operatorname{Hom}\left(C_{1}\left(\tilde{\Delta}, \tilde{\Sigma}_{u b}\left(q_{0}\right), \mathbb{R}\right)^{-}, \mathbb{C}\right)$. Since each 2simplex of $\Delta$ is a non-degenerate triangle, vectors $\left\{\left(\widetilde{\mathbf{u}}\left[q_{0}\right]+\widetilde{\mathbf{v}}\right)\left(e_{i}\right)\right\}_{i=1}^{3}$ also span a non-degenerate triangle when $\widetilde{\mathbf{v}}$ is sufficiently short. Collecting such new triangles defined from all 2-simplices of $\tilde{\Delta}$, and gluing them according to the combinatorial structure $\tilde{\Delta}$, we get a new singular Euclidean surface $\tilde{M}_{q_{0}}[\mathbf{v}]$ which is homeomorphic to $\tilde{M}_{q_{0}}$ by a piecewise affine mapping $\tilde{F}_{\mathbf{v}}: \tilde{M}_{q_{0}} \rightarrow \tilde{M}_{q_{0}}[\mathbf{v}]$ defined by assembling the 
affine deformations on the 2-simplices of $\tilde{\Delta}$. Since $i_{q_{0}}^{*}(\widetilde{\mathbf{v}})=-\widetilde{\mathbf{v}}, \tilde{M}_{q_{0}}[\mathbf{v}]$ admits an involution $i_{q_{0}}[\mathbf{v}]$ satisfies $i_{q_{0}}[\mathbf{v}] \circ \tilde{F}_{\mathbf{v}}=\tilde{F}_{\mathbf{v}} \circ i_{q_{0}}$, and the piecewise affine mapping $\tilde{F}_{\mathbf{v}}$ descends to a quasiconformal mapping (a piecewise affine mapping) $F_{\mathbf{v}}$ on $M_{0}$ to a Riemann surface $M_{0}[\mathbf{v}]$. The surface $\tilde{M}_{q_{0}}[\mathbf{v}]$ admits a $\Delta$-complex structure $\tilde{\Delta}[\mathbf{v}]$ inherited form $\tilde{\Delta}$ on $\tilde{M}_{q_{0}}$ which is equivariant under the action of the involution $i_{q_{0}}[\mathbf{v}]$. The $\Delta$-complex structure $\tilde{\Delta}[\mathbf{v}]$ descends to a $\Delta$-complex structure $\Delta[\mathbf{v}]$ on $M_{0}[\mathbf{v}]$.

Denote by $w$ the flat coordinate for $\tilde{M}_{q_{0}}[\mathbf{v}]$ (defined on each 2-simplex of $\tilde{\Delta}[\mathbf{v}]$ ). The holomorphic 1-form $d w$ on each 2-simplex for $\tilde{M}_{q_{0}}[\mathbf{v}]$ defines a holomorphic 1-form $\boldsymbol{\omega}_{q_{0}}[\mathbf{v}]$ on $\tilde{M}_{q_{0}}[\mathbf{v}]$. The square $\boldsymbol{\omega}_{q_{0}}[\mathbf{v}]^{2}$ descends to a holomorphic quadratic differential $\mathbf{q}\left[q_{0}, \mathbf{v}\right]$ on $M_{0}[\mathbf{v}]$.

Let $x_{\mathbf{v}}=\left(M_{0}[\mathbf{v}], F_{\mathbf{v}} \circ f_{0}\right) \in \mathcal{T}_{g, m}$. Since $\tilde{F}_{\mathbf{v}}$ sends the vertices of $\tilde{\Delta}$ to the vertices of $\tilde{\Delta}[\mathbf{v}]$, we can see that $\mathbf{q}\left[q_{0}, \mathbf{v}\right] \in \mathcal{Q}\left(\boldsymbol{\pi}\left(q_{0}\right)\right) \cap \mathcal{Q}_{x_{\mathbf{v}}}$ when $\widetilde{\mathbf{v}}$ is sufficiently short, $\mathbf{q}\left[q_{0}, 0\right]=q_{0}$, and

$$
\mathbf{u}\left[\mathbf{q}\left[q_{0}, \mathbf{v}\right]\right](C)=\int_{\left(\tilde{F}_{\mathbf{v}}\right)_{*}(C)} \boldsymbol{\omega}_{q_{0}}[\mathbf{v}]=\left(\mathbf{u}\left[q_{0}\right]+\mathbf{v}\right)(C)
$$

for all $C \in H_{1}\left(\tilde{M}_{q_{0}}, \tilde{\Sigma}_{u b}, \mathbb{R}\right)^{-}$. Summarizing the above argument, we get a commuting diagram

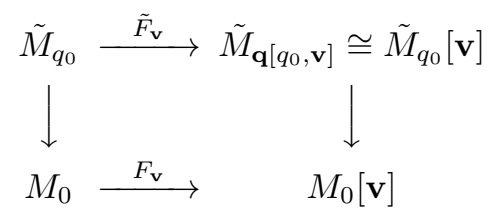

when the lift $\widetilde{\mathbf{v}} \in \operatorname{Hom}\left(C_{1}\left(\tilde{\Delta}, \tilde{\Sigma}_{u b}\left(q_{0}\right), \mathbb{R}\right)^{-}, \mathbb{C}\right)$ of $\mathbf{v} \in \operatorname{Hom}\left(q_{0}\right) \cong T_{q_{0}} \mathcal{Q}\left(\boldsymbol{\pi}\left(q_{0}\right)\right)$ is sufficiently short, where the vertical directions are double-branched coverings with covering involutions $i_{q_{0}}$ and $i_{q_{0}}[\mathbf{v}]$.

5.4. Teichmüller deformations. Let $x_{0}=\left(M_{0}, f_{0}\right) \in \mathcal{T}_{g, m}$ and $q_{0} \in \mathcal{Q}_{x_{0}}$. For $t \geq 0$, let $h_{t}: M_{0} \rightarrow M_{t}$ be the Teichmüller mapping associated to the Beltrami differential $\tanh (t) \overline{q_{0}} /\left|q_{0}\right|$ (cf. $(2.2 .2)$. Let $x_{t, q_{0}}=\left(M_{t}, h_{t} \circ f_{0}\right) \in \mathcal{T}_{g, m}$ and let $\mathbf{Q}\left[t, q_{0}\right] \in \mathcal{Q}_{x_{t, q_{0}}}$ be the terminal differential (e.g., [26]). For our purpose, we assume that the Teichmüller mapping $h_{t}$ is represented as an affine mapping associated to the matrix $\left(\begin{array}{cc}1 & 0 \\ 0 & e^{-2 t}\end{array}\right)$ in terms of the natural coordinates (distinguished parameters) of the initial and terminal differentials (cf. [57, Chapter II]). In particular, $\left\|\mathbf{Q}\left[t, q_{0}\right]\right\|=e^{-2 t}\left\|q_{0}\right\|$ and $v\left(\mathbf{Q}\left[t, q_{0}\right]\right)=v\left(q_{0}\right)$ from our assumption (cf. [24, Lemma 4.3] and (18) below). It is known that $\mathbf{Q}\left[t, q_{0}\right] \in \mathcal{Q}\left(\boldsymbol{\pi}\left(q_{0}\right)\right)$ for $t \geq 0$ (e.g., 38]).

The Teichmüller mapping $h_{t}$ lifts as a quasiconformal mapping $\tilde{h}_{t}: \tilde{M}_{q_{0}} \rightarrow \tilde{M}_{q_{t, q_{0}}}$ which is equivariant under the action of the involutions. The lift gives the identification $H_{1}\left(\tilde{M}_{\mathbf{Q}\left[t, q_{0}\right]}, \tilde{\Sigma}_{u b}\left(\mathbf{Q}\left[t, q_{0}\right]\right), \mathbb{R}\right)^{-} \cong H_{1}\left(\tilde{M}_{q_{0}}, \tilde{\Sigma}_{u b}\left(q_{0}\right), \mathbb{R}\right)^{-}$. By the analytic continuation along a continuous path $t \mapsto \mathbf{Q}\left[t, q_{0}\right] \in \mathcal{Q}\left(\boldsymbol{\pi}\left(q_{0}\right)\right)$ from initial point $q_{0}$, the chart given in Proposition 4.1 extends a neighborhood of the path. The image of $\mathbf{Q}\left[t, q_{0}\right]$ by the chart satisfies

$$
\begin{aligned}
\mathbf{u}\left[\mathbf{Q}\left[t, q_{0}\right]\right](C) & =\operatorname{Re}\left(\mathbf{u}\left[q_{0}\right](C)\right)+\sqrt{-1} e^{-2 t} \operatorname{Im}\left(\mathbf{u}\left[q_{0}\right](C)\right) \\
& =\frac{1+e^{-2 t}}{2} \mathbf{u}\left[q_{0}\right](C)+\frac{1-e^{-2 t}}{2} \overline{\mathbf{u}\left[q_{0}\right](C)}
\end{aligned}
$$

for $C \in H_{1}\left(\tilde{M}_{q_{0}}, \tilde{\Sigma}_{u b}\left(q_{0}\right), \mathbb{R}\right)^{-}$. 
5.5. Piecewise affine deformations around Teichmüller geodesics. Let $\mathbf{v} \in$ $\operatorname{Hom}\left(q_{0}\right) \cong T_{q_{0}} \mathcal{Q}\left(\boldsymbol{\pi}\left(q_{0}\right)\right)$ and $t>0$. When the lift $\widetilde{\mathbf{v}}$ is sufficiently short as in 5.3 . we define $x_{\mathbf{v}} \in \mathcal{T}_{g, m}$ and $\mathbf{q}\left[q_{0}, \mathbf{v}\right] \in \mathcal{Q}_{x_{\mathbf{v}}}$ associated to $\mathbf{v}$ (cf. 5.3 ). Consider the Teichmüller deformation on $x_{\mathbf{v}}$ associated to $\mathbf{q}\left[q_{0}, \mathbf{v}\right]$ and $t$. From the discussion in $\$ 5.4$, the chart in Proposition 4.1 is defined around the terminal differential $\mathbf{Q}\left[t, \mathbf{q}\left[q_{0}, \mathbf{v}\right]\right]$ when the lift of $\mathbf{v}$ is sufficiently short.

From (16) and (18), $\mathbf{Q}\left[t, \mathbf{q}\left[q_{0}, 0\right]\right]=\mathbf{Q}\left[t, q_{0}\right], \mathbf{Q}\left[0, \mathbf{q}\left[q_{0}, \mathbf{v}\right]\right]=\mathbf{q}\left[q_{0}, \mathbf{v}\right]$ and

$$
\mathbf{u}\left[\mathbf{Q}\left[t, \mathbf{q}\left[q_{0}, \mathbf{v}\right]\right]\right](C)=\operatorname{Re}\left(\left(\mathbf{u}\left[q_{0}\right]+\mathbf{v}\right)(C)\right)+\sqrt{-1} e^{-2 t} \operatorname{Im}\left(\left(\mathbf{u}\left[q_{0}\right]+\mathbf{v}\right)(C)\right)
$$

for $t \geq 0$ and $C \in H_{1}\left(\tilde{M}_{q_{0}}, \tilde{\Sigma}_{u b}\left(q_{0}\right), \mathbb{R}\right)^{-}$when the lift $\widetilde{\mathbf{v}}$ of $\mathbf{v} \in \operatorname{Hom}\left(q_{0}\right)$ is sufficiently short.

5.6. The hypercohomology group. Following Hubbard-Masur [24], we recall the description of the holomorphic tangent space $T_{q} \mathcal{Q}_{g, m}$ at $q \in \mathcal{Q}_{g, m}$ as the first hypercohomology group $\mathbb{H}^{1}\left(L^{\bullet}\right)$ a complex of sheaves (cf. [19] or [20]). We will need the Kodaira-Spencer identification of the tangent space of Teichmüller space with the first cohomology group of the sheaf of holomorphic vector fields (for instance, see [32; see also [26] and [27]).

Let $X$ and $q$ be a holomorphic vector field and a holomorphic quadratic differential on an open set of a Riemann surface $M$. Denote by $L_{X} q$ the Lie derivative of $q$ along $X$. Let $\Theta_{M}$ and $\Omega_{M}^{\otimes 2}$ be the sheaves of germs of holomorphic vector fields with zeros at marked points and meromorphic quadratic differentials on $M$ with (at most) first order poles at marked points, respectively.

Let $q_{0} \in \mathcal{Q}_{g, m}$ and $x_{0}=\left(M_{0}, f_{0}\right) \in \mathcal{T}_{g, m}$ with $q_{0} \in \mathcal{Q}_{x_{0}}$ ( $q_{0}$ need not be generic). The tangent space $T_{q_{0}} \mathcal{Q}_{g, m}$ is identified with the first hypercohomology group of the complex of sheaves

$$
L \bullet: \quad 0 \longrightarrow \Theta_{M_{0}} \stackrel{L \cdot q_{0}}{\longrightarrow} \Omega_{M_{0}}^{\otimes 2} \longrightarrow 0 .
$$

The first cochain group is the direct sum $C^{0}\left(M_{0}, \Omega_{M_{0}}^{\otimes 2}\right) \oplus C^{1}\left(M_{0}, \Theta_{M_{0}}\right)$. Consider an appropriate covering $\mathcal{U}=\left\{U_{i}\right\}_{i}$ on $M_{0}$ such that $\mathbb{H}^{1}\left(L^{\bullet}\right) \cong \mathbb{H}^{1}\left(\mathcal{U}, L^{\bullet}\right)$ (see the proof of [24, Proposition 4.5]).

A cochain $\left(\left\{\phi_{i}\right\}_{i},\left\{X_{i j}\right\}_{i, j}\right)$ in $C^{0}\left(\mathcal{U}, \Omega_{M_{0}}^{\otimes 2}\right) \oplus C^{1}\left(\mathcal{U}, \Theta_{M_{0}}\right)$ is said to be a cocycle if it satisfies

$$
\delta\left\{X_{i j}\right\}_{i, j}=X_{i j}+X_{j k}+X_{k i}=0, \quad \delta\left\{\phi_{i}\right\}_{i}=\phi_{i}-\phi_{j}=L_{X_{i j}}\left(q_{0}\right) .
$$

A coboundary is a cochain $\left(\left\{\phi_{i}\right\}_{i},\left\{X_{i j}\right\}_{i, j}\right)$ of the form

$$
X_{i j}=Z_{i}-Z_{j}=\delta\left\{Z_{i}\right\}_{i}, \quad \phi_{i}=L_{Z_{i}}\left(q_{0}\right)
$$

for some 0-cochain $\left\{Z_{i}\right\}_{i} \in C^{0}\left(\mathcal{U}, \Theta_{M_{0}}\right)$ (cf. Figure 3).

For the hypercohomology class $\left[\left(\left\{\phi_{i}\right\}_{i},\left\{X_{i j}\right\}_{i, j}\right)\right] \in \mathbb{H}^{1}\left(L^{\bullet}\right)$, when the KodairaSpencer class of the 1-cochain $\left\{X_{i j}\right\}_{i, j}$ is trivial in $H^{1}\left(M_{0}, \Theta_{M_{0}}\right)$, the hypercohomology class $\left[\left(\left\{\phi_{i}\right\}_{i},\left\{X_{i j}\right\}_{i, j}\right)\right]$ is associated to a holomorphic quadratic differential on $M_{0}$. Indeed, from (20) and (21),

$$
\phi_{i}-\phi_{j}=L_{X_{i j}}\left(q_{0}\right)=L_{Z_{i}}\left(q_{0}\right)-L_{Z_{j}}\left(q_{0}\right)
$$

and $\left\{\phi_{i}-L_{Z_{i}}\left(q_{0}\right)\right\}_{i}$ defines a holomorphic quadratic differential on $M_{0}$. 


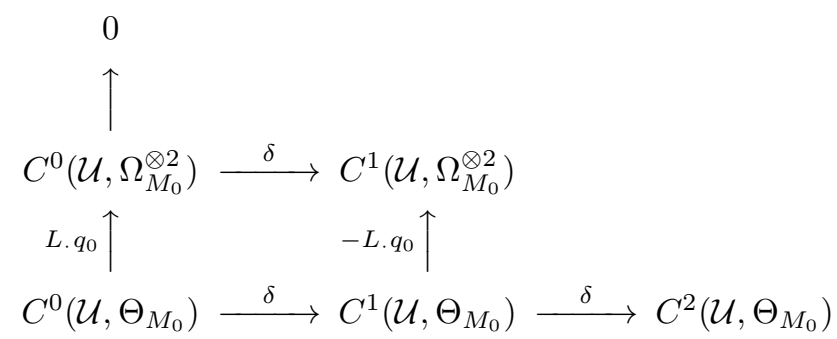

Figure 3. Double complex for the tangent spaces to $\mathcal{Q}_{g, m}$.

5.7. Homomorphisms and hypercohomology classes. From Proposition 4.1, we have a canonical inclusion

$$
\operatorname{Hom}\left(q_{0}\right) \cong T_{q_{0}} \mathcal{Q}(\boldsymbol{\pi}(q)) \hookrightarrow T_{q_{0}} \mathcal{Q}_{g, m} \cong \mathbb{H}^{1}\left(L^{\bullet}\right) .
$$

Let $\mathbf{v} \in \operatorname{Hom}\left(q_{0}\right)$ and $\left[\left(\left\{\phi_{i}\right\}_{i},\left\{X_{i j}\right\}_{i, j}\right)\right] \in \mathbb{H}^{1}\left(L^{\bullet}\right)$ be the corresponding hypercohomology class via (22). Take a 0 -cochain $\left\{X_{i}\right\}_{i}$ of the sheaf of $C^{\infty}$-vector fields such that $X_{i}-X_{j}=X_{i j}$ on $U_{i} \cap U_{j}$, and each $X_{i}$ vanishes at any marked point of $M_{0}$. The 1-cochain $\left\{X_{i j}\right\}_{i, j}$ defines a holomorphic tangent vector at $x_{0}$ associated to the infinitesimal Beltrami differential $-\left(X_{i}\right)_{\bar{z}}$ on $M_{0}$ (cf. [46, (3.6)]). The minus sign comes from our " $i, j$-convention" in the definition of the hypercohomology (compare with Equation (7.27) in [26, §7.2.4]). The holomorphic tangent vector from the 1-cochain $\left\{X_{i j}\right\}_{i, j}$ coincides with the image of $\mathbf{v} \in \operatorname{Hom}\left(q_{0}\right)\left(\hookrightarrow T_{q_{0}} \mathcal{Q}_{g, m}\right)$ via the differential of the projection $\mathcal{Q}_{g, m} \rightarrow \mathcal{T}_{g, m}$.

After choosing the covering $\mathcal{U}=\left\{U_{i}\right\}_{i}$ appropriately, the right and left sides of the inclusion (22) is related to the following formula:

$$
\mathbf{v}(C)=\int_{C} \Omega\left[q_{0}, \mathbf{v}\right]
$$

for $C \in H_{1}\left(\tilde{M}_{q_{0}}, \tilde{\Sigma}_{u b}\left(q_{0}\right), \mathbb{R}\right)^{-}$, where $\Omega\left[q_{0}, \mathbf{v}\right]$ is a $C^{\infty}$-closed 1 -form on $\tilde{M}_{q_{0}}$ defined by

$$
\boldsymbol{\Omega}\left[q_{0}, \mathbf{v}\right]=\left(\frac{\tilde{\phi}_{i}}{2 \omega_{q_{0}}}-\omega_{q_{0}}^{\prime} \tilde{X}_{i}-\omega_{q_{0}}\left(\tilde{X}_{i}\right)_{z}\right) d z-\omega_{q_{0}}\left(\tilde{X}_{i}\right)_{\bar{z}} d \bar{z}
$$

on $U_{i}$, and tildes in (24) mean objects (differentials or vector fields, etc.) on the covering space $\tilde{M}_{q_{0}}$ which are obtained as lifts of objects on $M_{0}$. For a proof, see e.g., [46, Lemma 3.1]. Actually, in [46, Lemma 3.1], we discuss only in the case where $q_{0}$ is generic. However, we can deduce (24) the same argument since we consider deformations of holomorphic quadratic differentials along strata as seen in the discussion in [14, Lemma 5.6].

Remark 5.1. We notice the following:

(1) Fix $\epsilon>0$ sufficiently small. Then, $\phi_{i}$ is the $\lambda$-derivative of the infinitesimal deformation of a holomorphic mapping $\{|\lambda|<\epsilon\} \ni \lambda \mapsto \mathbf{q}\left[q_{0}, \lambda \mathbf{v}\right] \epsilon$ $\mathcal{Q}\left(\boldsymbol{\pi}\left(q_{0}\right)\right)$ on $U_{i}$ at $\lambda=0$ (e.g., [46, §3.3]). Since $\mathbf{q}\left[q_{0}, \lambda \mathbf{v}\right]$ varies in $\mathcal{Q}\left(\boldsymbol{\pi}\left(q_{0}\right)\right)$, we can see that $\boldsymbol{o}_{p}\left(\phi_{i}\right) \geq \boldsymbol{o}_{p}\left(q_{0}\right)-1$ for $p \in \Sigma_{s \backslash m}\left(q_{0}\right) \cap U_{i}$ and $\boldsymbol{o}_{p}\left(\phi_{i}\right) \geq$ $\boldsymbol{o}_{p}\left(q_{0}\right)$ for $p \in \Sigma_{s m}\left(q_{0}\right) \cap U_{i}$ (cf. [14, Lemma 5.2]. Hence the first term of the coefficient of $d z$ of the differential $\boldsymbol{\Omega}\left[q_{0}, \mathbf{v}\right]$ in (24) is holomorphic around $\tilde{\Sigma}\left(q_{0}\right) \cap U_{i}$. 
(2) For $\mathbf{v} \in \operatorname{Hom}\left(q_{0}\right)$, we define the complex conjugate $\overline{\mathbf{v}} \in \operatorname{Hom}\left(q_{0}\right)$ of $\mathbf{v}$ by

$$
\overline{\mathbf{v}}(C)=\overline{\mathbf{v}(C)}
$$

for $C \in H_{1}\left(\tilde{M}_{q_{0}}, \tilde{\Sigma}_{u b}\left(q_{0}\right), \mathbb{R}\right)^{-}$. We can easily deduce from (23) that

$$
\int_{C} \boldsymbol{\Omega}\left[q_{0}, \overline{\mathbf{v}}\right]=\int_{C} \overline{\boldsymbol{\Omega}\left[q_{0}, \mathbf{v}\right]}
$$

for $C \in H_{1}\left(\tilde{M}_{q_{0}}, \tilde{\Sigma}_{u b}\left(q_{0}\right), \mathbb{R}\right)^{-}$. Notice that the complex conjugate of $\mathbf{v}$ here is thought of as a tangent vector in the $(1,0)$-part in the complexification of the real tangent vector space at $q_{0}$. Compare [31, Proposition 1.5 in Chapter IX].

We claim the following (cf. [13] and [14, Lemma 5.6]).

Proposition 5.1. Let $q_{0} \in \mathcal{Q}_{g, m}$. Let $\mathbf{v} \in \operatorname{Hom}\left(q_{0}\right)$ and $\left.\left[\left\{\phi_{i}\right\}_{i},\left\{X_{i j}\right\}_{i, j}\right)\right]$ be the corresponding hypercohomology class. When the Kodaira-Spencer class of $\left\{X_{i j}\right\}_{i, j}$ is trivial,

$$
\boldsymbol{\Omega}\left[q_{0}, \mathbf{v}\right]=\frac{\pi_{q_{0}}^{*}(\psi)}{\omega_{q_{0}}}
$$

for some $\psi \in \mathcal{Q}_{x_{0}}^{T}\left(q_{0}\right)$.

Proof. The assumption implies that there is a 0 -cochain $\left\{Z_{i}\right\}_{i} \in C^{0}\left(\mathcal{U}, \Theta_{M_{0}}\right)$ such that $Z_{i}-Z_{j}=X_{i j}$. From (24) we have

$$
\boldsymbol{\Omega}\left[q_{0}, \mathbf{v}\right]=\left(\frac{\tilde{\phi}_{i}}{2 \omega_{q_{0}}}-\omega_{q_{0}}^{\prime} \tilde{Z}_{i}-\omega_{q_{0}} \tilde{Z}_{i}^{\prime}\right) d z=\frac{\tilde{\psi}_{i}}{2 \omega_{q_{0}}} d z
$$

where

$$
\psi_{i}=\phi_{i}-L_{Z_{i}}\left(q_{0}\right)=\phi_{i}-\left(q_{0}^{\prime} Z_{i}+2 q_{0} Z_{i}^{\prime}\right) .
$$

As discussed in the last paragraph of $\$ 5.6,\left\{\psi_{i}\right\}_{i}$ defines a holomorphic quadratic differential $\psi$ on $M_{0}$. We can check from (1) in Remark 5.1 and (27) that $\psi \in$ $\mathcal{Q}_{x_{0}}^{T}\left(q_{0}\right)$.

Let $x_{0}=\left(M_{0}, f_{0}\right) \in \mathcal{T}_{g, m}$. Suppose $q_{0} \in \mathcal{Q}_{x_{0}}$ is generic. The projection $\Pi: \mathcal{Q}_{g, m} \rightarrow \mathcal{T}_{g, m}$ induces a complex linear map

$$
T_{q_{0}} \mathcal{Q}_{g, m} \cong \operatorname{Hom}\left(q_{0}\right) \ni \mathbf{v} \mapsto \mathrm{v}\left(\mathbf{v}, q_{0}\right):=\left.d \Pi\right|_{q_{0}}[\mathbf{v}] \in T_{x_{0}} \mathcal{T}_{g, m}
$$

via the differential.

Proposition 5.2 (Hodge-Kodaira decomposition). Under the above notation, we have

$$
\mathbf{v}(C)=\int_{C} \frac{\pi_{q_{0}}^{*}\left(\eta_{\mathrm{v}\left(\overline{\mathbf{v}}, q_{0}\right)}\right)}{\omega_{q_{0}}}+\int_{C} \overline{\left(\frac{\pi_{q_{0}}^{*}\left(\eta_{\mathrm{v}\left(\mathbf{v}, q_{0}\right)}\right)}{\omega_{q_{0}}}\right)}
$$

for $C \in H_{1}\left(\tilde{M}_{q_{0}}, \mathbb{R}\right)^{-}=H_{1}\left(\tilde{M}_{q_{0}}, \tilde{\Sigma}_{u b}\left(q_{0}\right), \mathbb{R}\right)^{-}$.

Proof. From the definition of the $q_{0}$-realizations, for $\phi \in \mathcal{Q}_{x_{0}}$,

$$
\begin{aligned}
\int_{M_{0}} \mu \phi & =\int_{M_{0}} \frac{\overline{\eta_{\mathrm{v}\left(\mathbf{v}, q_{0}\right)}}}{\left|q_{0}\right|} \phi=\frac{1}{2} \int_{\tilde{M}_{q_{0}}} \frac{\overline{\pi_{q_{0}}^{*}\left(\eta_{\mathrm{v}\left(\mathbf{v}, q_{0}\right)}\right)}}{\left|\omega_{q_{0}}\right|^{2}} \pi_{q_{0}}^{*}(\phi) \\
& =-\frac{\sqrt{-1}}{4} \int_{\tilde{M}_{q_{0}}} \overline{\left(\frac{\pi_{q_{0}}^{*}\left(\eta_{\mathrm{v}\left(\mathbf{v}, q_{0}\right)}\right)}{\omega_{q_{0}}}\right)} \wedge \frac{\pi_{q_{0}}^{*}(\phi)}{\omega_{q_{0}}} .
\end{aligned}
$$


Let $\boldsymbol{\Omega}\left[q_{0}, \mathbf{v}\right]^{(0,1)}$ be the $(0,1)$-part of $\boldsymbol{\Omega}\left[q_{0}, \mathbf{v}\right]$. From (24), a Beltrami differential $\boldsymbol{\Omega}\left[q_{0}, \mathbf{v}\right]^{(0,1)} / \omega_{q_{0}}$ on $\tilde{M}_{q_{0}}$ is the lift of the infinitesimal Beltrami differential $\mu$ on $M_{0}$ associated to $\mathrm{v}\left(\mathbf{v}, q_{0}\right)$. Let $\boldsymbol{\Omega}^{h}+\overline{\mathbf{\Omega}^{a h}}$ be the harmonic form in the de Rham cohomology class of $\boldsymbol{\Omega}\left[q_{0}, \mathbf{v}\right]$, where $\boldsymbol{\Omega}^{h}$ and $\boldsymbol{\Omega}^{a h}$ are holomorphic 1-forms on $\tilde{M}_{q_{0}}$. Then,

$$
\begin{aligned}
\int_{M_{0}} \mu \phi & =\frac{1}{2} \int_{\tilde{M}_{q_{0}}} \frac{\boldsymbol{\Omega}\left[q_{0}, \mathbf{v}\right]^{(0,1)}}{\omega_{q_{0}}} \pi_{q_{0}}^{*}(\phi)=-\frac{\sqrt{-1}}{4} \int_{\tilde{M}_{q_{0}}} \boldsymbol{\Omega}\left[q_{0}, \mathbf{v}\right] \wedge \frac{\pi_{q_{0}}^{*}(\phi)}{\omega_{q_{0}}} \\
& =-\frac{\sqrt{-1}}{4} \int_{\tilde{M}_{q_{0}}} \overline{\boldsymbol{\Omega}^{a h}} \wedge \frac{\pi_{q_{0}}^{*}(\phi)}{\omega_{q_{0}}} .
\end{aligned}
$$

We can easily check that every holomorphic 1-form in the $(-1)$-eigenspace of the space of holomorphic 1 -forms is presented as $\pi_{q_{0}}^{*}(\phi) / \omega_{q_{0}}$ for some $\phi \in \mathcal{Q}_{x_{0}}$. From (28) and (29), we have $\boldsymbol{\Omega}^{a h}=\pi_{q_{0}}^{*}\left(\eta_{\mathrm{v}\left(\mathrm{v}, q_{0}\right)}\right) / \omega_{q_{0}}$. Since the harmonic differential in the de Rham cohomology class is unique, from (25), we deduce that $\boldsymbol{\Omega}^{h}=$ $\pi_{q_{0}}^{*}\left(\eta_{\mathrm{v}\left(\overline{\mathbf{v}}, q_{0}\right)}\right) / \omega_{q_{0}}$.

For a generic differential $q_{0} \in \mathcal{Q}_{g, m}$, we define

$$
\operatorname{Hom}_{0}\left(q_{0}\right)=\left\{\mathbf{v} \in \operatorname{Hom}\left(q_{0}\right) \mid \mathbf{v}\left(\mathbf{v}, q_{0}\right)=0\right\} .
$$

From Proposition 5.2, we have the following.

Corollary 5.1. Let $x_{0} \in \mathcal{T}_{g, m}$ and $q_{0} \in \mathcal{Q}_{x_{0}}$ be a generic differential. Then, the mapping

$$
\operatorname{Hom}_{0}\left(q_{0}\right) \ni \mathbf{v} \mapsto \eta_{\mathrm{v}\left(\overline{\mathbf{v}}, q_{0}\right)} \in \mathcal{Q}_{x_{0}}
$$

is a complex linear isomorphism.

Example 5.1. For generic $q \in \mathcal{Q}_{g, m}$,

$$
\int_{C} \frac{\pi_{q}^{*}(q)}{\omega_{q}}=\int_{C} \omega_{q}=\mathbf{u}[q](C)=\int_{C} \frac{\pi_{q}^{*}\left(\eta_{\mathrm{v}}(\overline{\mathbf{u}[q]}, q)\right.}{\omega_{q}}+\overline{\left(\frac{\pi_{q}^{*}\left(\eta_{\mathrm{v}(\mathbf{u}[q], q)}\right)}{\omega_{q}}\right)}
$$

for $C \in H_{1}\left(\tilde{M}_{q}, \mathbb{R}\right)^{-}$. Therefore, $\eta_{\mathrm{v}(\overline{\mathbf{u}[q]}, q)}=q$ and $\eta_{\mathrm{v}(\mathbf{u}[q], q)}=0$.

\section{Stratification of Teichmüller SPACE}

6.1. Stratifications on $\mathcal{Q}_{x_{0}}$. Let $x_{0} \in \mathcal{T}_{g, m}$. Dumas 14 defined a stratification of $\mathcal{Q}_{x_{0}}$ by symbols applying the Whitney stratification (cf. [54] and 60]). The stratification on $\mathcal{Q}_{g, m}$ provides a stratification on $\mathcal{Q}_{x_{0}}$ by complex analytic sets. This stratification on $\mathcal{Q}_{x_{0}}$ can be refined as a complex analytic stratification $\left\{Z_{i}\right\}_{i \in I}=\left\{Z_{i, x_{0}}\right\}_{i \in I}$ satisfying the following conditions:

(1) Each $Z_{i}$ is a complex submanifold of $\mathcal{Q}_{x_{0}}-\{0\}$ invariant under the action of $\mathbb{C}^{*}$;

(2) the symbol is constant on each stratum $Z_{i}$;

(3) $\mathcal{Q}_{x_{0}}-\{0\}=\bigsqcup_{i \in I} Z_{i}$;

(4) $Z_{i} \cap Z_{j}=\emptyset$ if $i \neq j$; and

(5) the closure $Z_{j}$ is a complex analytic set, and if $Z_{i} \cap \overline{Z_{j}} \neq \emptyset$ for $i, j \in I$, then $Z_{i} \subset \overline{Z_{j}}$. 
The refinement refers to changing the stratification in such a way that each new stratum is entirely contained in one of the old strata. Under the situation in the above (5), $\operatorname{dim}_{\mathbb{C}} Z_{i}<\operatorname{dim}_{\mathbb{C}} Z_{j}$ if $Z_{i} \neq Z_{j}$. There is a unique stratum $Z_{\infty}$ consisting of all generic differentials (we assume the index set $I$ contains a symbol " $\infty$ "). Since the stratification is locally finite, we have the following.

Lemma 6.1. For any $q_{0} \in Z_{i}-\{0\}$, there is a neighborhood $U$ in $\mathcal{Q}_{x_{0}}-\{0\}$ of $q_{0}$ such that $I_{U}=\left\{i \in I \mid Z_{i} \cap U \neq \emptyset\right\}$ is a finite set; and if $Z_{j} \cap U \neq \emptyset$, then $Z_{i} \cap \overline{Z_{j}} \neq \emptyset$.

We extend an observation by Dumas as follows (cf. Lemma 5.2 in [14).

Proposition 6.1 (Tangent space to the strata in fibers). Let $\left\{Z_{i}\right\}_{i \in I}$ be the stratification of $\mathcal{Q}_{y_{0}}$ defined in $₫ 6.1$. Let $q_{0} \in Z_{i}$. If we identify the tangent space $T_{q_{0}} Z_{i}$ as a subspace of $\mathcal{Q}_{x_{0}}$, we have $T_{q_{0}} Z_{i} \subset \mathcal{Q}_{x_{0}}^{T}\left(q_{0}\right)$.

Proof. Let $p_{0} \in \Sigma_{s}\left(q_{0}\right)$ and $k_{0}=\boldsymbol{o}_{p_{0}}\left(q_{0}\right)$. For simplicity, $q_{0}$ is assumed to be represented as $q_{0}=z^{k_{0}} d z^{2}$ around $p_{0}$ with the coordinate $z$ with $z\left(p_{0}\right)=0$. From the universal deformation of the singularities, the deformation of $q_{0}$ around $p_{0}$ is described as the Lie derivative

$$
L_{X}\left(z^{k_{0}} d z^{2}\right)=\left(k_{0} z^{k_{0}-1} X(z)+2 z^{k_{0}} X^{\prime}(z)\right) d z^{2}
$$

along a holomorphic vector field $X=X(z)(\partial / \partial z)$ around $p_{0}$, where $X(0)=0$ if $p_{0} \in \Sigma_{m}\left(q_{0}\right)$ (cf. [24, Proposition 3.1]; see also Lemma 5.2 in [14]). One can see that the infinitesimal deformation $\dot{q}$ satisfies

$$
\boldsymbol{o}_{p_{0}}(\dot{q}) \geq \begin{cases}k_{0}-1 & \left(\text { if } p_{0} \in \Sigma_{s \backslash m}\left(q_{0}\right)\right), \\ k_{0} & \left(\text { if } p_{0} \in \Sigma_{s m}\left(q_{0}\right)\right),\end{cases}
$$

and is contained in $\mathcal{Q}_{x_{0}}^{T}\left(q_{0}\right)$.

6.2. Stratification of Teichmüller space. Let $x_{0} \in \mathcal{T}_{g, m}$. Let $\left\{Z_{i}\right\}_{i \in I}$ be the stratification of $\mathcal{Q}_{x_{0}}-\{0\}$ defined in 6 . Let $\mathcal{U} \mathcal{Q}_{x_{0}}$ be the unit ball in $\mathcal{Q}_{x_{0}}$ with respect to the $L^{1}$-norm and let $\Xi_{x_{0}}: \mathcal{U} \mathcal{Q}_{x_{0}} \rightarrow \mathcal{T}_{g, m}$ be the Teichmüller homeomorphism discussed in $\$ 2.2 .2$. For $i \in I$, we define $\mathcal{T}_{i}=\Xi_{x_{0}}\left(Z_{i} \cap \mathcal{U} \mathcal{Q}_{x_{0}}\right)$. The purpose of this section is to show the following.

Theorem 3 (Stratification). The collection $\left\{\mathcal{T}_{i}\right\}_{i \in I}$ is a stratification of realanalytic submanifolds in $\mathcal{T}_{g, m}-\left\{x_{0}\right\}$.

Since $\Xi_{x_{0}}$ is a homeomorphism, $\left\{\mathcal{T}_{i}\right\}_{i \in I}$ is a stratification of topological manifolds in $\mathcal{T}_{g, m}-\left\{x_{0}\right\}$. Namely, each $\mathcal{T}_{i}$ is a locally closed topological submanifold of $\mathcal{T}_{g, m}-\{0\}$, the collection $\left\{\mathcal{T}_{i}\right\}_{i \in I}$ is locally finite and satisfies

(3') $\mathcal{T}_{g, m}-\{0\}=\bigsqcup_{i \in I} \mathcal{T}_{i}$;

(4') $\mathcal{T}_{i} \cap \mathcal{T}_{j}=\emptyset$ if $i \neq j$; and

(5') if $\mathcal{T}_{i} \cap \overline{\mathcal{T}_{j}} \neq \emptyset$ for $i, j \in I$, then $\mathcal{T}_{i} \subset \overline{\mathcal{T}_{j}}$.

(The numbers correspond to those in the properties of complex analytic stratifications given in 6.1 ) We will show that the restriction of $\Xi_{x_{0}}$ to each $Z_{i} \cap \mathcal{U} \mathcal{Q}_{x_{0}}$ is a real-analytic immersion. The author does not know if the closure $\overline{\mathcal{T}_{i}}$ is a realanalytic subset of $\mathcal{T}_{g, m}-\{0\}$ for each $i \in I$. Notice that Theorem 3 is recognized as a kind of refinement of Masur's result [39, Proposition 2.2]. 
Proof of Theorem 3. Let $i \in I$. Notice from the definition that $Z_{i}$ is a complex submanifold of $\mathcal{Q}_{x_{0}}$. From (5) and 5 5.4, the Teichmüller homeomorphism $\Xi_{x_{0}}$ on $Z_{i}$ is described as

$$
\Xi_{x_{0}}(q)=\Pi\left(\mathbf{Q}\left[\tanh ^{-1}(\|q\|), q\right]\right)
$$

for $q \in Z_{i} \cap \mathcal{U} \mathcal{Q}_{x_{0}}$. From Proposition 4.1 and Riemann's formula, the norm $Z_{i} \ni$ $q \mapsto\|q\|$ varies real-analytically (cf. [34, §1] and [16, Chapter III]). Hence the mapping

$$
Z_{i} \ni q \mapsto \mathbf{Q}\left[\tanh ^{-1}(\|q\|), q\right] \in \mathcal{Q}\left(\boldsymbol{\pi}\left(q_{0}\right)\right)
$$

is real-analytic. Therefore, $\Xi_{x_{0}}$ is also real-analytic on $Z_{i}$ since $\Pi$ is holomorphic. Hence, to complete the proof, it suffices to show that the (real) differential of the restriction of $\Xi_{x_{0}}$ to $Z_{i}$ is non-singular.

Let $q_{0} \in Z_{i} \cap \mathcal{U} \mathcal{Q}_{x_{0}}$ and $\mathbf{v} \in \operatorname{Hom}\left(q_{0}\right)\left(\hookrightarrow T_{q_{0}} \mathcal{Q}\left(\boldsymbol{\pi}\left(q_{0}\right)\right)\right)$ which is tangent to $Z_{i}$. For simplicity, set $Q_{0}=\mathbf{Q}\left[\tanh ^{-1}\left(\left\|q_{0}\right\|\right), q_{0}\right]$ and $x_{1}=\left(M_{1}, f_{1}\right)=x_{\tanh ^{-1}\left(\left\|q_{0}\right\|\right), q_{0}} \in$ $\mathcal{T}_{g, m}$ (cf. \$5.4). From Proposition 6.1, there is $\psi \in \mathcal{Q}_{x_{0}}^{T}\left(q_{0}\right)$ such that

$$
\mathbf{v}(C)=\int_{C} \frac{\pi_{q_{0}}^{*}(\psi)}{\omega_{q_{0}}}
$$

for $C \in H_{1}\left(\tilde{M}_{q_{0}}, \tilde{\Sigma}_{u b}\left(q_{0}\right), \mathbb{R}\right)^{-}$.

Let $f:\{|\lambda|<\epsilon\} \rightarrow Z_{i}$ be a holomorphic disk with $q(0)=q_{0}$ and $q^{\prime}(0)=\mathbf{v}$. For simplicity, set $K(t)=\exp \left(2 \tanh ^{-1}(\|q(t)\|)=(1+\|q(t)\|) /(1-\|q(t)\|)\right.$ for $t \in \mathbb{R}$ with $|t|<\epsilon$. From (19) and (30),

$$
\begin{aligned}
& \left.\frac{d}{d t}\right|_{t=0} \mathbf{u}\left[\mathbf{Q}\left[\tanh ^{-1}(\|q(t)\|), q(t)\right]\right](C) \\
& =\operatorname{Re} \int_{C} \frac{\pi_{q_{0}}^{*}(\psi)}{\omega_{q_{0}}}+\sqrt{-1} \operatorname{Im}\left(K(0)^{-1} \int_{C} \frac{\pi_{q_{0}}^{*}(\psi)}{\omega_{q_{0}}}+\left.\left(K^{-1}\right)^{\prime}\right|_{t=0} \int_{C} \omega_{q_{0}}\right)
\end{aligned}
$$

for $C \in H_{1}\left(\tilde{M}_{q_{0}}, \tilde{\Sigma}_{u b}\left(q_{0}\right), \mathbb{R}\right)^{-}$. We denote by $\mathbf{w}(C)$ the right-hand side of (31).

Recall that the holomorphic tangent space is the $(1,0)$-part of the complexification of the real tangent vector space (cf. [31, Chapter IX]). In general, for a complex manifold with a local chart $z=\left(z_{1}, \cdots, z_{n}\right)$, a holomorphic tangent vector $\sum_{j=1}^{n} a_{j}\left(\partial / \partial z_{j}\right)$ is the $(1,0)$-part of a real tangent vector $\sum_{j=1}^{n}\left(a_{j}\left(\partial / \partial z_{j}\right)+\right.$ $\left.\overline{a_{j}}\left(\partial / \partial \bar{z}_{j}\right)\right)$ of the underlying differential structure. The variation (31) stands for the $(1,0)$-part of the image of the corresponding real tangent vector to $\mathbf{v}$ under the (real) differential of the map $Z_{i} \ni q \mapsto \mathbf{Q}\left[\tanh ^{-1}(\|q\|), q\right] \in \mathcal{Q}\left(\boldsymbol{\pi}\left(q_{0}\right)\right)$ around $q_{0}$. Then, w stands for a homomorphism in $\operatorname{Hom}\left(Q_{0}\right)\left(\subset T_{Q_{0}} \mathcal{Q}\left(\boldsymbol{\pi}\left(q_{0}\right)\right)\right)$ via the isomorphism $H_{1}\left(\tilde{M}_{q_{0}}, \tilde{\Sigma}_{u b}\left(q_{0}\right), \mathbb{R}\right)^{-} \cong H_{1}\left(\tilde{M}_{Q_{0}}, \tilde{\Sigma}_{u b}\left(Q_{0}\right), \mathbb{R}\right)^{-}$induced by the Teichmüller mapping from $x_{0}$ to $x_{1}$.

Suppose that the derivative

$$
\{|t|<\epsilon \mid t \in \mathbb{R}\} \ni t \mapsto \Pi\left(\mathbf{Q}\left[\tanh ^{-1}(\|q(t)\|), q(t)\right]\right) \in \mathcal{T}_{g, m}
$$

at $t=0$ vanishes. We will conclude $\mathbf{v}=0$.

Since $\Pi$ is holomorphic, the differential of $\Pi$ sends the (1,0)-part $T_{Q_{0}} \mathcal{Q}_{g, m}$ of the complexification of the real tangent space at $Q_{0}$ to that at $x_{1}$. From the assumption,

$$
\mathrm{v}\left(\mathbf{w}, Q_{0}\right)=\left.d \Pi\right|_{Q_{0}}[\mathbf{w}]=0
$$


in $T_{x_{1}} \mathcal{T}_{g, m}$ (cf. [31, Proposition 2.9, Chapter IX]). From Proposition 5.1, there is $\phi \in \mathcal{Q}_{x_{1}}^{T}\left(Q_{0}\right)$ such that

$$
\mathbf{w}(C)=\int_{C} \frac{\pi_{Q_{0}}^{*}(\phi)}{\omega_{Q_{0}}}
$$

for $C \in H_{1}\left(\tilde{M}_{q_{0}}, \tilde{\Sigma}_{u b}\left(q_{0}\right), \mathbb{R}\right)^{-} \cong H_{1}\left(\tilde{M}_{Q_{0}}, \tilde{\Sigma}_{u b}\left(Q_{0}\right), \mathbb{R}\right)^{-}$.

Let $\psi^{\prime} \in \mathcal{Q}_{x_{0}}$ and $\phi^{\prime} \in \mathcal{Q}_{x_{1}}$ be the holomorphic quadratic differentials defined by descending the squares $\left(\pi_{q_{0}}^{*}(\psi) / \omega_{q_{0}}\right)^{2}$ and $\left(\pi_{Q_{0}}^{*}(\phi) / \omega_{Q_{0}}\right)^{2}$, respectively. Comparing the real parts of (31) and (32) we have $v\left(\phi^{\prime}\right)=v\left(\psi^{\prime}\right)$ in $\mathcal{M F}$ (cf. 24, Lemma 4.3]). Since $K(0)=e^{2 d_{T}\left(x_{0}, x_{1}\right)}$,

$$
K(0)^{-1}\left\|\psi^{\prime}\right\|=e^{-2 d_{T}\left(x_{0}, x_{1}\right)} \operatorname{Ext}_{x_{0}}\left(v\left(\psi^{\prime}\right)\right) \leq \operatorname{Ext}_{x_{1}}\left(v\left(\phi^{\prime}\right)\right)=\left\|\phi^{\prime}\right\|
$$

from the Kerckhoff formula (see also [18, Lemma 4.1]).

By Riemann's formula, (12) and (31),

$$
\begin{aligned}
&\left\|\phi^{\prime}\right\|= \frac{1}{2} \int_{\tilde{M}_{Q_{0}}} \operatorname{Re}\left(\frac{\pi_{Q_{0}}^{*}(\phi)}{\omega_{Q_{0}}}\right) \wedge \operatorname{Im}\left(\frac{\pi_{Q_{0}}^{*}(\phi)}{\omega_{Q_{0}}}\right) \\
&= K(0)^{-1} \frac{1}{2} \int_{\tilde{M}_{q_{0}}} \operatorname{Re}\left(\frac{\pi_{q_{0}}^{*}(\psi)}{\omega_{q_{0}}}\right) \wedge \operatorname{Im}\left(\frac{\pi_{q_{0}}^{*}(\psi)}{\omega_{q_{0}}}\right) \\
& \quad+\left.\left(K^{-1}\right)^{\prime}\right|_{t=0} \frac{1}{2} \int_{\tilde{M}_{q_{0}}} \operatorname{Re}\left(\frac{\pi_{q_{0}}^{*}(\psi)}{\omega_{q_{0}}}\right) \wedge \operatorname{Im}\left(\omega_{q_{0}}\right) \\
&=K(0)^{-1}\left\|\psi^{\prime}\right\|+\left(K^{-1}\right)^{\prime}(0) \operatorname{Re}\left\{\frac{\sqrt{-1}}{4} \int_{\tilde{M}_{q_{0}}} \frac{\pi_{q_{0}}^{*}(\psi)}{\omega_{q_{0}}} \wedge \frac{\omega_{q_{0}}}{\omega}\right\} .
\end{aligned}
$$

Since

$$
\left.\frac{d\|q(t)\|}{d t}\right|_{t=0}=\operatorname{Re}\left\{\frac{\sqrt{-1}}{2} \int_{\tilde{M}_{q_{0}}} \frac{\pi_{q_{0}}^{*}(\psi)}{\omega_{q_{0}}} \wedge \overline{\omega_{q_{0}}}\right\},
$$

from (33) and (34), we obtain

$$
\begin{aligned}
0 & \leq\left\|\phi^{\prime}\right\|-K(0)^{-1}\left\|\psi^{\prime}\right\|=\left(K^{-1}\right)^{\prime}(0) \operatorname{Re}\left\{\frac{\sqrt{-1}}{4} \int_{\tilde{M}_{q_{0}}} \frac{\pi_{q_{0}}^{*}(\psi)}{\omega_{q_{0}}} \wedge \overline{\omega_{q_{0}}}\right\} \\
& =-\frac{1}{\left(1+\left\|q_{0}\right\|\right)^{2}}\left(\left.\frac{d\|q(t)\|}{d t}\right|_{t=0}\right)^{2},
\end{aligned}
$$

and $\left(K^{-1}\right)^{\prime}=0$ at $t=0$. Therefore, $\left\|\phi^{\prime}\right\|=K(0)^{-1}\left\|\psi^{\prime}\right\|$ from (34) again. Hence, we have $v\left(\psi^{\prime}\right)=s v\left(q_{0}\right)$ and $\psi^{\prime}=s^{2} q_{0}$ for some $s \geq 0$ from the uniqueness of the extremal problem for the Kerckhoff formula (or the Teichmüller uniqueness theorem; see [26, Theorem 5.9] or [1, $\S(3.5)]$ ). From (35), we obtain

$$
0=\left.\frac{d\|q(t)\|}{d t}\right|_{t=0}=s^{2}\left\|q_{0}\right\|
$$

and $s=0$. Therefore, $\psi^{\prime}=0$ and $\mathbf{v}=0$ from (30).

From (5), we conclude the following.

Corollary 6.1. The Teichmüller distance function $\mathcal{T}_{g, m}-\left\{x_{0}\right\} \ni x \mapsto d_{T}\left(x_{0}, x\right)$ is real-analytic on each stratum of $\left\{\mathcal{T}_{i}\right\}_{i \in I}$. 
Recall that the top stratum $Z_{\infty}$ of the stratification of $\mathcal{Q}_{x_{0}}$ is an open set which consists of generic differentials. From Theorem 3 the restriction of the Teichmüller homeomorphism $\Xi_{x_{0}}: Z_{\infty} \rightarrow \mathcal{T}_{\infty}$ is a real-analytic diffeomorphism. Hence, Corollary 6.1 is thought of as an extension of an observation by Rees in [52, $\S 2.3]$.

6.3. Proof of Theorem 2, We use the following removable singularity theorem due to Blanchet and Chirka.

Proposition 6.2 (Blanchet [3] and Chirka [8]). Let $\Omega$ be a domain in $\mathbb{C}^{N}$ and let $V \subset \Omega$ be a $C^{1}$-real submanifold with positive real codimension. Let $u$ be a function of class $C^{1}$ on $\Omega$. Then, $u$ is plurisubharmonic on $\Omega$ if so is $u$ on $\Omega-V$.

Indeed, Blanchet proved the above removable singularity theorem under the additional assumption that $u$ is of class $C^{2}$ on $\Omega-V$. However, the condition is eliminated by applying Chirka's theorem [8] with a standard argument with mollifiers. For completeness, we will confirm Proposition 6.2 in the appendix (99).

We return to our setting. Let $\left\{\mathcal{T}_{i}\right\}_{i \in I}$ be the stratification in Theorem 3 , Let $u$ be a function of class $C^{1}$ on $\mathcal{T}_{g, m}-\left\{x_{0}\right\}$ which is bounded above around $x_{0}$. Suppose that $u$ is plurisubharmonic on the top stratum $\mathcal{T}_{\infty}$.

Let $x_{1} \in \mathcal{T}_{g, m}-\left\{x_{0}\right\}$ and let $\mathcal{T}_{i}$ be the stratum containing $x_{1}$. Suppose that $u$ is extended as a plurisubharmonic function on $\mathcal{T}_{j}$ for all $j \in I$ with $\operatorname{dim} \mathcal{T}_{j}>\operatorname{dim} \mathcal{T}_{i}$. From the locally finiteness of the stratification, there is a small neighborhood $U$ of $x_{1}$ such that $I(U)=\left\{j \in I \mid \mathcal{T}_{j} \cap U \neq \emptyset\right\}$ is a finite set and $\mathcal{T}_{i} \cap \overline{\mathcal{T}_{j}} \neq \emptyset$ for $j \in I(U)$ from Lemma 6.1. From the assumption, $u$ is plurisubharmonic on $U-\mathcal{T}_{i}$. Since $\mathcal{T}_{i}$ is a real-analytic submanifold of $\mathcal{T}_{g, m}$ with positive codimension, by Proposition 6.2, $u$ is plurisubharmonic on $U$. This inductive procedure guarantees that $u$ is plurisubharmonic on $\mathcal{T}_{g, m}-\left\{x_{0}\right\}$. Since $u$ is bounded above around $x_{0}, u$ is extended as a pluriharmonic function on $\mathcal{T}_{g, m}$ (cf. [30, Theorem 2.9.22]).

\section{Pluricomplex Green function on the Teichmüller space}

In this section, we will show the following theorem which implies Theorem 1. since the Teichmüller distance is the Kobayashi distance on $\mathcal{T}_{g, m}$ (cf. [53] and (7.1).

Theorem 4 (Plurisubharmonicity). Let $x_{0} \in \mathcal{T}_{g, m}$. The log-tanh of the Teichmüller distance function

$$
\mathcal{T}_{g, m} \ni x \mapsto u_{x_{0}}(x):=\log \tanh d_{T}\left(x_{0}, x\right)
$$

is plurisubharmonic on $\mathcal{T}_{g, m}$.

Earle [15] showed that $u_{x_{0}}$ is of class $C^{1}$ on $\mathcal{T}_{g, m}-\left\{x_{0}\right\}$. Since $u_{x_{0}}(x) \rightarrow-\infty$ as $x \rightarrow x_{0}$, from Theorem 2 it suffices to show that $u_{x_{0}}$ is plurisubharmonic on the top stratum $\mathcal{T}_{\infty}$, which will be proved in Theorem 5 in $\$ 7.6$.

7.1. Backgrounds from complex analysis. Let $X$ be a complex manifold. Let $p \in X$ and $z=\left(z_{1}, \cdots, z_{n}\right)$ be a holomorphic local chart around $p$. Let $u$ be a $C^{2}$ function around $p$ on $X$. For $v=\sum_{i=1}^{n} v_{i}\left(\partial / \partial z_{i}\right) \in T_{p} X$, we define the Levi form of $u$ by

$$
\mathcal{L}(u)[v, \bar{v}]=\sum_{i, j=1}^{n} \frac{\partial^{2} u}{\partial z_{i} \partial \bar{z}_{j}}(z(p)) v_{i} \overline{v_{j}}
$$


Let $g:\{\lambda \in \mathbb{C}|| \lambda \mid<\epsilon\} \rightarrow X$ be a holomorphic mapping with $g(0)=p$ and $g_{*}(\partial / \partial \lambda)=v$. Then, we see

$$
\mathcal{L}(u)[v, \bar{v}]=\frac{\partial^{2}(u \circ g)}{\partial \lambda \partial \bar{\lambda}}(0) .
$$

A $C^{2}$-function $u$ on $X$ is called plurisubharmonic if $\mathcal{L}(u)[v, \bar{v}] \geq 0$ for $v \in T_{p} X$ and $p \in X$. In general, a function $u$ on a domain $\Omega$ on $\mathbb{C}^{N}$ is called plurisubharmonic if for any $a \in \Omega$ and $b \in \mathbb{C}^{N}, \lambda \mapsto u(a+\lambda b)$ is subharmonic or identically $-\infty$ on every component of $\{\lambda \in \mathbb{C} \mid a+\lambda b \in \Omega\}$.

A bounded domain $\Omega$ in $\mathbb{C}^{N}$ is said to be hyperconvex if it admits a negative continuous plurisubharmonic exhaustion (cf. [56]). Krushkal' [35] showed that Teichmüller space is hyperconvex (see also [46]).

Demailly [10 observed that for any bounded hyperconvex domain $\Omega$ in $\mathbb{C}^{n}$ and $w \in \Omega$, there is a unique plurisubharmonic function $g_{\Omega, w}: \Omega \rightarrow[-\infty, 0)$ such that

(1) $\left(d d^{c} g_{\Omega, w}\right)^{n}=(2 \pi)^{n} \delta_{w}$, where $\delta_{w}$ is the Dirac measure with support at $w$; and

(2) $g_{\Omega, w}(z)=\sup _{v}\{v(z)\}$ where the supremum runs over all non-positive plurisubharmonic function $v$ on $\Omega$ with $v(z) \leq \log \|z-w\|+O(1)$ around $z=w$

(cf. 10, Théorème 4.3]). The function $g_{\Omega}(w, z)=g_{\Omega, w}(z)$ is called the pluricomplex Green function on $\Omega$. The pluricomplex Green function was introduced by Klimek 29]. Klimek showed that

$$
\log \tanh \operatorname{Car}_{\Omega}(z, w) \leq g_{\Omega}(z, w) \leq \log \tanh \operatorname{Kob}_{\Omega}(z, w)
$$

for $z, w \in \Omega$ and in the second inequality in (39), the equality holds if the third term of (39) is plurisubharmonic, where $\mathrm{Car}_{\Omega}$ and $\mathrm{Kob}_{\Omega}$ are the Carathéodory distance and the Kobayashi distance on $\Omega$, respectively (cf. [29, Corollaries 1.2 and 1.4]).

7.2. Setting. Let $q_{0} \in Z_{\infty} \cap \mathcal{Q}_{x_{0}}$ and $x_{1}=\Xi_{x_{0}}\left(q_{0}\right)$. Let $v \in T_{x_{1}} \mathcal{T}_{g, m}$ and $g:\{|\lambda|<$ $\epsilon\} \rightarrow \mathcal{T}_{\infty}$ be a holomorphic mapping with $g(0)=0$ and $g_{*}\left(\partial /\left.\partial \lambda\right|_{\lambda=0}\right)=v$. For simplicity, let

$$
\begin{aligned}
q_{\lambda} & =\Xi_{x_{0}}^{-1}(g(\lambda)) \in Z_{\infty} \subset \mathcal{Q}_{x_{0}}, \\
Q_{\lambda} & =\mathbf{Q}\left[\tanh ^{-1}\left(\left\|q_{\lambda}\right\|\right), q_{\lambda}\right] \in \mathcal{Q}_{g(\lambda)}, \\
d(\lambda) & =d_{T}\left(x_{0}, g(\lambda)\right), \text { and } d_{0}=d(0)=d_{T}\left(x_{0}, x_{1}\right)
\end{aligned}
$$

for $\lambda \in\{|\lambda|<\epsilon\}$. Notice again that each $Q_{\lambda}$ is generic since the Teichmüller mapping preserves the order of singular points. For calculations later, we notice from the definition that

$$
\left\|q_{\lambda}\right\|=\tanh (d(\lambda)) \quad \text { and } \quad\left\|Q_{\lambda}\right\|=e^{-2 d(\lambda)}\left\|q_{\lambda}\right\| .
$$

7.2.1. We define $\mathbf{v}_{1}, \mathbf{v}_{2} \in \operatorname{Hom}\left(q_{0}\right)\left(\cong T_{q_{0}} \mathcal{Q}_{g, m}\right)$ by

$$
\mathbf{u}\left[q_{\lambda}\right]=\mathbf{u}\left[q_{0}\right]+\lambda \mathbf{v}_{1}+\bar{\lambda} \mathbf{v}_{2}+o(|\lambda|)
$$

as $\lambda \rightarrow 0$ on $H_{1}\left(\tilde{M}_{q_{0}}, \mathbb{R}\right)^{-} \cong H_{1}\left(\tilde{M}_{q_{0}}, \tilde{\Sigma}_{u b}\left(q_{0}\right), \mathbb{R}\right)^{-}$. Since $q_{\lambda} \in \mathcal{Q}_{x_{0}}$ for all $\lambda$, we deduce $\mathrm{v}\left(\mathbf{v}_{i}, q_{0}\right)=0$ for $i=1,2$ and $\mathbf{v}_{1}, \mathbf{v}_{2} \in \operatorname{Hom}_{0}\left(q_{0}\right)\left(\cong \mathcal{Q}_{x_{0}} \subset T_{q_{0}} \mathcal{Q}_{g, m}\right)$. We will use the notation

$$
\mathrm{D}_{x_{1}}(v)=\mathbf{v}_{1} \quad \text { and } \quad \overline{\mathrm{D}}_{x_{1}}(v)=\mathbf{v}_{2}
$$


after calculating the first derivative and the Levi form of the Teichmüller distance (cf. $\$ 7.5)$. However, in the following calculation, we will use the notation $\mathbf{v}_{1}$ and $\mathbf{v}_{2}$ for simplicity.

From (18) and (41),

$$
\begin{aligned}
\mathbf{u}\left[Q_{\lambda}\right] & =\operatorname{Re}\left(\mathbf{u}\left[q_{\lambda}\right]\right)+\sqrt{-1} e^{-2 d(\lambda)} \operatorname{Im}\left(\mathbf{u}\left[q_{\lambda}\right]\right) \\
& =\mathbf{u}\left[q_{0}\right]+\lambda \mathbf{w}_{1}+\bar{\lambda} \mathbf{w}_{2}+o(|\lambda|)
\end{aligned}
$$

on $H_{1}\left(\tilde{M}_{q_{0}}, \mathbb{R}\right)^{-}$as $\lambda \rightarrow 0$, where $\mathbf{w}_{1}$ and $\mathbf{w}_{2}$ are in $\operatorname{Hom}\left(q_{0}\right)$ defined by

$$
\left\{\begin{array}{l}
\mathbf{w}_{1}=\frac{1+e^{-2 d_{0}}}{2} \mathbf{v}_{1}+\frac{1-e^{-2 d_{0}}}{2} \overline{\mathbf{v}_{2}}-d_{\lambda} e^{-2 d_{0}}\left(\mathbf{u}\left[q_{0}\right]-\overline{\mathbf{u}\left[q_{0}\right]}\right), \\
\mathbf{w}_{2}=\frac{1-e^{-2 d_{0}}}{2} \overline{\mathbf{v}_{1}}+\frac{1+e^{-2 d_{0}}}{2} \mathbf{v}_{2}-\overline{d_{\lambda}} e^{-2 d_{0}}\left(\mathbf{u}\left[q_{0}\right]-\overline{\mathbf{u}\left[q_{0}\right]}\right),
\end{array}\right.
$$

where $d_{\lambda}$ is the $\lambda$-derivative of the Teichmüller distance function $d(\lambda)$ at $\lambda=0$. In (43), we canonically identify $\operatorname{Hom}\left(Q_{0}\right)$ with $\operatorname{Hom}\left(q_{0}\right)$, and $\mathbf{w}_{1}$ and $\mathbf{w}_{2}$ stands for tangent vectors in $T_{Q_{0}} \mathcal{Q}_{g, m} \cong \operatorname{Hom}\left(Q_{0}\right)$, while the right-hand sides of (43) are tangent vectors in $\operatorname{Hom}\left(q_{0}\right) \cong T_{q_{0}} \mathcal{Q}_{g, m}$. See the discussion in the proof of Theorem 3 and (2) of Remark 5.1

Since $\Pi\left(\mathbf{u}\left[Q_{\lambda}\right]\right)=g(\lambda)$, we have

$$
\mathrm{v}\left(\mathbf{w}_{1}, Q_{0}\right)=v \quad \text { and } \quad \mathrm{v}\left(\mathbf{w}_{2}, Q_{0}\right)=0
$$

and $\mathbf{w}_{2} \in \operatorname{Hom}_{0}\left(Q_{0}\right)$, since $\Pi$ is holomorphic.

7.2.2. Since $H_{1}\left(\tilde{M}_{q_{0}}, \mathbb{R}\right)^{-} \cong H_{1}\left(\tilde{M}_{q_{0}}, \tilde{\Sigma}_{u b}\left(q_{0}\right), \mathbb{R}\right)^{-}, \operatorname{Hom}\left(q_{0}\right)$ is canonically isomorphic to the cohomology group $H^{1}\left(\tilde{M}_{q_{0}}, \mathbb{R}\right)^{-}$. We define the wedge product $\wedge$ on $\operatorname{Hom}\left(q_{0}\right)$ by

$$
\mathbf{x} \wedge \mathbf{y}=\int_{\tilde{M}_{q_{0}}} \frac{\pi_{q_{0}}^{*}\left(\eta_{\mathrm{v}\left(\overline{\mathbf{x}}, q_{0}\right)}\right)}{\omega_{q_{0}}} \wedge \overline{\left(\frac{\pi_{q_{0}}^{*}\left(\eta_{\mathrm{v}\left(\mathbf{y}, q_{0}\right)}\right)}{\omega_{q_{0}}}\right)}-\frac{\pi_{q_{0}}^{*}\left(\eta_{\mathrm{v}\left(\overline{\mathbf{y}}, q_{0}\right)}\right)}{\omega_{q_{0}}} \wedge \overline{\left(\frac{\pi_{q_{0}}^{*}\left(\eta_{\mathrm{v}\left(\mathbf{x}, q_{0}\right)}\right)}{\omega_{q_{0}}}\right)}
$$

for $\mathbf{x}, \mathbf{y} \in \operatorname{Hom}\left(q_{0}\right)$. Then

$$
\sqrt{-1} \mathbf{u}[q] \wedge \overline{\mathbf{u}[q]}=4\|q\|
$$

(cf. (5) and Example 5.11).

Example 7.1 (Teichmüller disk). The Teichmüller disk associated to $q_{0}$ is defined as an isometric holomorphic disk in $\mathcal{T}_{g, m}$ defined by the holomorphic family of Beltrami differentials

$$
\mathbb{D} \ni \lambda \mapsto\left(\frac{\lambda+\tanh \left(d_{0}\right)}{1+\tanh \left(d_{0}\right) \lambda}\right) \frac{\overline{q_{0}}}{\left|q_{0}\right|}
$$

on $M_{0}$. With the Teichmüller homeomorphism (4), the Teichmüller disk is described as

$$
\mathbb{D} \ni \lambda \mapsto \Xi_{x_{0}}\left(\left(\frac{\bar{\lambda}+\tanh \left(d_{0}\right)}{1+\tanh \left(d_{0}\right) \bar{\lambda}}\right) \frac{q_{0}}{\left\|q_{0}\right\|}\right)
$$


For $\lambda \in \mathbb{D}$. let $\mathbf{D}_{q_{0}}(\lambda)$ be the right-hand side of (47). By definition, $\mathbf{D}_{q_{0}}(0)=x_{1}$ and $\mathbf{D}_{q_{0}}\left(-\tanh \left(d_{0}\right)\right)=x_{0}$. Then

$$
\begin{aligned}
\mathbf{u}\left[\Xi_{x_{0}}^{-1}\left(\mathbf{D}_{q_{0}}(\lambda)\right)\right] & =\frac{1}{\left\|q_{0}\right\|^{1 / 2}}\left(\frac{\bar{\lambda}+\tanh \left(d_{0}\right)}{1+\tanh \left(d_{0}\right) \bar{\lambda}}\right)^{1 / 2} \mathbf{u}\left[q_{0}\right] \\
& =\mathbf{u}\left[q_{0}\right]+\frac{\bar{\lambda}}{\sinh \left(2 d_{0}\right)} \mathbf{u}\left[q_{0}\right]+o(|\lambda|),
\end{aligned}
$$

where the branch of the square root is taken to be $1^{1 / 2}=1$.

7.3. The first variation of the Teichmüller distance. We give the first variational formula of the Teichmüller distance function in our setting. From Riemann's formula, (40) and (46), we deduce

$$
\begin{aligned}
d_{\lambda} & =\left.\left(\tanh ^{-1}\left(\left\|q_{\lambda}\right\|\right)\right)_{\lambda}\right|_{\lambda=0}=\frac{1}{1-\left\|q_{0}\right\|^{2}} \frac{\sqrt{-1}}{4}\left(\mathbf{v}_{1} \wedge \overline{u\left[q_{0}\right]}+u\left[q_{0}\right] \wedge \overline{\mathbf{v}_{2}}\right) \\
& =\frac{\sqrt{-1} \cosh ^{2}\left(d_{0}\right)}{4}\left(\mathbf{v}_{1} \wedge \overline{u\left[q_{0}\right]}+u\left[q_{0}\right] \wedge \overline{\mathbf{v}_{2}}\right) .
\end{aligned}
$$

On the other hand, Earle [15] gave the first variational formula

$$
d_{\lambda}=\frac{1}{2\left\|Q_{0}\right\|} \int_{M_{1}} \mu Q_{0}
$$

where $\mu$ is the infinitesimal Beltrami differential on $M_{1}$ representing $v$. From (40), (44), and (46),

$$
\begin{aligned}
& d_{\lambda}=\frac{1}{2\left\|Q_{0}\right\|} \int_{M_{1}} \mu Q_{0}=\frac{1}{2 e^{-2 d_{0} \tanh \left(d_{0}\right)}} \int_{M_{1}} \frac{\overline{\eta_{v}}}{\left|Q_{0}\right|} Q_{0} \\
& =\frac{e^{2 d_{0}}}{2 \tanh \left(d_{0}\right)} \frac{-\sqrt{-1}}{4} \int_{\tilde{M}_{Q_{0}}}\left(\frac{\pi_{Q_{0}}^{*}\left(\eta_{\mathrm{v}\left(\mathbf{w}_{1}, Q_{0}\right)}\right)}{\omega_{Q_{0}}}\right) \wedge \omega_{Q_{0}}=\frac{-\sqrt{-1} e^{2 d_{0}}}{8 \tanh \left(d_{0}\right)} \mathbf{w}_{1} \wedge \mathbf{u}\left[Q_{0}\right] \\
& =\frac{-\sqrt{-1} e^{2 d_{0}}}{8 \tanh \left(d_{0}\right)}\left(\frac{1+e^{-2 d_{0}}}{2} \mathbf{v}_{1}+\frac{1-e^{-2 d_{0}}}{2} \overline{\mathbf{v}_{2}}-d_{\lambda} e^{-2 d_{0}}\left(\mathbf{u}\left[q_{0}\right]-\overline{\mathbf{u}\left[q_{0}\right]}\right)\right) \\
& =\frac{-\sqrt{-1} e^{2 d_{0}}}{8 \tanh \left(d_{0}\right)}\left(\frac{1-e^{-4 d_{0}}}{4} \overline{\mathbf{v}_{2}} \wedge \mathbf{u}\left[q_{0}\right]+d_{\lambda} e^{-2 d_{0}} \frac{1+e^{-2 d_{0}}}{2} \overline{\mathbf{u}\left[q_{0}\right]} \wedge \mathbf{u}\left[q_{0}\right]\right. \\
& \left.\quad+\frac{1-e^{-4 d_{0}}}{2} \mathbf{v}_{1} \wedge \overline{\mathbf{u}\left[q_{0}\right]}-d_{\lambda} e^{-2 d_{0}} \frac{1-e^{-2 d_{0}}}{2} \mathbf{u}\left[q_{0}\right] \wedge \overline{\mathbf{u}\left[q_{0}\right]}\right) \\
& =-\frac{\sqrt{-1} \cosh ^{2}\left(d_{0}\right)}{8}\left(\mathbf{v}_{1} \wedge \overline{\mathbf{u}\left[q_{0}\right]}-\mathbf{u}\left[q_{0}\right] \wedge \overline{\mathbf{v}_{2}}\right)+\frac{\sqrt{-1} d_{\lambda}}{8 \tanh \left(d_{0}\right)} \mathbf{u}\left[q_{0}\right] \wedge \overline{\mathbf{u}\left[q_{0}\right]} \\
& =-\frac{\sqrt{-1} \cosh ^{2}\left(d_{0}\right)}{8}\left(\mathbf{v}_{1} \wedge \overline{\mathbf{u}\left[q_{0}\right]}-\mathbf{u}\left[q_{0}\right] \wedge \overline{\mathbf{v}_{2}}\right)+\frac{d_{\lambda}}{2} .
\end{aligned}
$$

Therefore, we obtain

$$
d_{\lambda}=\frac{\sqrt{-1} \cosh ^{2}\left(d_{0}\right)}{4}\left(-\mathbf{v}_{1} \wedge \overline{\mathbf{u}\left[q_{0}\right]}+\mathbf{u}\left[q_{0}\right] \wedge \overline{\mathbf{v}_{2}}\right) .
$$

Thus, from (49) and (51) we conclude the following. 
Lemma 7.1 (First variational formula). Under the notation in $\$ 7.2$, we have

$$
d_{\lambda}=\frac{\sqrt{-1} \cosh ^{2}\left(d_{0}\right)}{4} \mathbf{u}\left[q_{0}\right] \wedge \overline{\mathbf{v}_{2}}
$$

and $\mathbf{v}_{1} \wedge \overline{\mathbf{u}\left[q_{0}\right]}=0$.

7.4. Levi form of $d_{T}$. Let $u\left[q_{\lambda}\right]_{\lambda} \in \operatorname{Hom}\left(q_{0}\right)$ be the $\lambda$-derivative of the family $\left\{\mathbf{u}\left[q_{\lambda}\right]\right\}_{|\lambda|<\epsilon}$ of the representation. Namely, $u\left[q_{\lambda}\right]_{\lambda}(C)=\left(u\left[q_{\lambda}\right](C)\right)_{\lambda}$ for $C \in$ $H_{1}\left(\tilde{M}_{q_{0}}, \mathbb{R}\right)^{-}$. Notice from the notation in 87.2 that $u\left[q_{\lambda}\right]_{\lambda}=\mathbf{v}_{1}$ at $\lambda=0$. We also define $u\left[q_{\lambda}\right]_{\bar{\lambda}}$ and $u\left[q_{\lambda}\right]_{\lambda \bar{\lambda}}$ in the same manner. From Lemma 7.1] the $\lambda$-derivative of $d(\lambda)=d_{T}\left(x_{0}, g(\lambda)\right)$ on a disk $\{|\lambda|<\epsilon\}$ is rewritten as

$$
d_{\lambda}(\lambda)=\frac{\sqrt{-1} \cosh ^{2}(d(\lambda))}{4} \mathbf{u}\left[q_{\lambda}\right] \wedge \overline{\mathbf{u}\left[q_{\lambda}\right]_{\bar{\lambda}}} .
$$

Therefore,

$$
\begin{aligned}
d_{\lambda \bar{\lambda}}(0)= & \frac{\sqrt{-1}}{4} \cdot 2 \sinh \left(d_{0}\right) \cosh \left(d_{0}\right) d_{\bar{\lambda}} \cdot \mathbf{u}\left[q_{0}\right] \wedge \overline{\mathbf{v}_{2}} \\
& +\frac{\sqrt{-1} \cosh ^{2}\left(d_{0}\right)}{4} \mathbf{v}_{2} \wedge \overline{\mathbf{v}_{2}}+\frac{\sqrt{-1} \cosh ^{2}\left(d_{0}\right)}{4} \mathbf{u}\left[q_{0}\right] \wedge \overline{\left.\mathbf{u}\left[q_{0}\right]_{\lambda \bar{\lambda}}\right|_{\lambda=0}}
\end{aligned}
$$

From Lemma 7.1 again,

$$
0=\left(\mathbf{u}\left[q_{\lambda}\right] \wedge \overline{\mathbf{u}\left[q_{\lambda}\right]_{\lambda}}\right)_{\lambda}=\mathbf{u}\left[q_{\lambda}\right]_{\lambda} \wedge \overline{\mathbf{u}\left[q_{\lambda}\right]_{\lambda}}+\mathbf{u}\left[q_{\lambda}\right] \wedge \overline{\mathbf{u}\left[q_{\lambda}\right]_{\lambda \bar{\lambda}}}
$$

Thus, the Laplacian $d_{\lambda \bar{\lambda}}(0)$ of the distance function $d(\lambda)$ at $\lambda=0$ is

$$
\frac{\cosh ^{3}\left(d_{0}\right) \sinh \left(d_{0}\right)}{8}\left|\mathbf{u}\left[q_{0}\right] \wedge \overline{\mathbf{v}_{2}}\right|^{2}+\frac{\sqrt{-1} \cosh ^{2}\left(d_{0}\right)}{4}\left(\mathbf{v}_{2} \wedge \overline{\mathbf{v}_{2}}-\mathbf{v}_{1} \wedge \overline{\mathbf{v}_{1}}\right) .
$$

7.5. Complex tangent spaces of the spheres. We use the notation (42). Notice that

$$
\begin{aligned}
& T_{x_{1}} \mathcal{T}_{g, m} \ni v \mapsto \mathrm{D}_{x_{1}}(v) \in \operatorname{Hom}_{0}\left(q_{0}\right), \\
& T_{x_{1}} \mathcal{T}_{g, m} \ni v \mapsto \overline{\mathrm{D}}_{x_{1}}(v) \in \operatorname{Hom}_{0}\left(q_{0}\right),
\end{aligned}
$$

are complex and anti-complex linear, respectively.

From (38), Lemma 7.1, and (53), the first derivative and the Levi form of the Teichmüller distance function $\mathcal{T}_{g, m} \ni x \mapsto d_{T}\left(x_{0}, x\right)$ at $x_{1} \in \mathcal{T}_{g, m}-\left\{x_{0}\right\}$ are rewritten as

$$
\begin{aligned}
\partial d_{T}\left(x_{0}, \cdot\right)[v] & =\frac{\sqrt{-1} \cosh ^{2}\left(d_{0}\right)}{4} \mathbf{u}\left[q_{0}\right] \wedge \overline{\overline{\mathrm{D}}_{x_{1}}(v)} \\
\mathcal{L}\left(d_{T}\left(x_{0}, \cdot\right)\right)[v, \bar{v}] & =\frac{\cosh ^{3}\left(d_{0}\right) \sinh \left(d_{0}\right)}{8}\left|\mathbf{u}\left[q_{0}\right] \wedge \overline{\overline{\mathrm{D}}_{x_{1}}(v)}\right|^{2} \\
& +\frac{\sqrt{-1} \cosh ^{2}\left(d_{0}\right)}{4}\left(\overline{\mathrm{D}}_{x_{1}}(v) \wedge \overline{\overline{\mathrm{D}}_{x_{1}}(v)}-\mathrm{D}_{x_{1}}(v) \wedge \overline{\mathrm{D}_{x_{1}}(v)}\right)
\end{aligned}
$$

for $v \in T_{x_{1}} \mathcal{T}_{g, m}$. For $v_{1}, v_{2} \in T_{x_{1}} \mathcal{T}_{g, m}$, the Hermitian form of the Levi form is represented as

$$
\begin{aligned}
\mathcal{L}\left(d_{T}\left(x_{0}, \cdot\right)\right)\left[v_{1}, \overline{v_{2}}\right] & =\frac{\cosh ^{3}\left(d_{0}\right) \sinh \left(d_{0}\right)}{8} \mathbf{u}\left[q_{0}\right] \wedge \overline{\overline{\mathrm{D}}_{x_{1}}\left(v_{1}\right)} \cdot \overline{\mathbf{u}\left[q_{0}\right]} \wedge \overline{\mathrm{D}}_{x_{1}}\left(v_{2}\right) \\
& +\frac{\sqrt{-1} \cosh ^{2}\left(d_{0}\right)}{4}\left(\overline{\mathrm{D}}_{x_{1}}\left(v_{2}\right) \wedge \overline{\overline{\mathrm{D}}_{x_{1}}\left(v_{1}\right)}-\mathrm{D}_{x_{1}}\left(v_{1}\right) \wedge \overline{\mathrm{D}_{x_{1}}\left(v_{2}\right)}\right)
\end{aligned}
$$

from (54). 
For $r>0$, we consider the sphere $S\left(x_{0}, r\right)=\left\{x \in \mathcal{T}_{g, m} \mid d_{T}\left(x_{0}, x\right)=r\right\}$ of the Teichmüller distance. For $x_{1} \in S\left(x_{0}, r\right)$, we define

$$
\begin{aligned}
H_{x_{1}}^{1,0}=H_{x_{1}}^{1,0}\left(S\left(x_{0}, r\right)\right) & =\left\{v \in T_{x_{1}} \mathcal{T}_{g, m} \mid \partial d_{T}\left(x_{0}, \cdot\right)[v]=0\right\} \\
& =\left\{v \in T_{x_{1}} \mathcal{T}_{g, m} \mid \mathbf{u}\left[q_{0}\right] \wedge \overline{\overline{\mathrm{D}}_{x_{1}}(v)}=0\right\} .
\end{aligned}
$$

The subspace $H_{x_{1}}^{1,0}$ is the $(1,0)$-part of the complex tangent space of the sphere $S\left(x_{0}, r\right)$ (cf. [4, $\left.\S 7\right]$ ). Let $\boldsymbol{\nu}\left(x_{1}\right) \in T_{x_{1}} \mathcal{T}_{g, m}$ be the tangent vector associated to the infinitesimal Beltrami differential $\overline{Q_{0}} /\left|Q_{0}\right|$ where $Q_{0}$ is the terminal differential of the Teichmüller mapping from $x_{0}$ to $x_{1}$. The vector $\boldsymbol{\nu}\left(x_{1}\right) \in T_{x_{1}} \mathcal{T}_{g, m}$ is tangent to the Teichmüller disk passing $x_{0}$ and $x_{1}$. From (48),

$$
\mathrm{D}_{x_{1}}\left(\boldsymbol{\nu}\left(x_{1}\right)\right)=0, \quad \overline{\mathrm{D}}_{x_{1}}\left(\boldsymbol{\nu}\left(x_{1}\right)\right)=\frac{1}{\sinh \left(2 d_{0}\right)} \mathbf{u}\left[q_{0}\right] .
$$

Proposition 7.1 (CR tangent space). The complex tangent space $H_{x_{1}}^{1,0}$ is perpendicular to $\boldsymbol{\nu}\left(x_{1}\right)$ with respect to the Levi form of the Teichmüller distance.

Proof. From (55) and (56), $\mathcal{L}\left(d_{T}\left(x_{0}, \cdot\right)\right)\left[v, \overline{\boldsymbol{\nu}\left(x_{1}\right)}\right]=0$ for $v \in H_{x_{1}}^{1,0}$.

Lemma 7.2 (Non-negativity on $H^{1,0}$ ). For $v \in H_{x_{1}}^{1,0}$,

$$
\sqrt{-1}\left(\overline{\mathrm{D}}_{x_{1}}(v) \wedge{\overline{\mathrm{D}_{x_{1}}}}(v)-\mathrm{D}_{x_{1}}(v) \wedge \overline{\mathrm{D}_{x_{1}}(v)}\right) \geq 0 .
$$

Proof. From [46, Corollary 1.2], the Teichmüller distance function $\mathcal{T}_{g, m} \ni x \mapsto$ $d_{T}\left(x_{0}, x\right)$ is plurisubharmonic (see also [36, Corollary 3]). Hence the Levi form of the distance function is non-negative on $H_{x_{1}}^{1,0}$. The assertion follows from (54).

7.6. Log-tanh of $d_{T}$ is plurisubharmonic on $\mathcal{T}_{\infty}$. We set

$$
u_{x_{0}}(x)=\log \tanh \left(d_{T}\left(x_{0}, x\right)\right)
$$

for $x \in \mathcal{T}_{g, m}$. From (5),

$$
u_{x_{0}}\left(\Xi_{x_{0}}(q)\right)=\log \|q\|
$$

for $q \in \mathcal{U} \mathcal{Q}_{x_{0}}$. Recall that $\mathcal{T}_{\infty}$ is the top stratum of the stratification of $\mathcal{T}_{g, m}-\left\{x_{0}\right\}$ which is obtained in Theorem 3 .

Theorem 5 (Plurisubharmonicity). $u_{x_{0}}$ is plurisubharmonic on $\mathcal{T}_{\infty}$.

Proof. Let $x_{1} \in \mathcal{T}_{\infty}$ and $q_{0} \in \mathcal{T}_{x_{0}}$ with $x_{1}=\Xi_{x_{0}}\left(q_{0}\right)$ as above. Since $u_{x_{0}}(g(\lambda))=$ $\log \left\|q_{\lambda}\right\|$ for $\lambda \in\{|\lambda|<\epsilon\}$, from the direct calculation, the Levi form of $u_{x_{0}}$ is given as

$$
\begin{gathered}
\mathcal{L}\left(u_{x_{0}}\right)[v, \bar{v}]=\frac{\sqrt{-1}}{4 \tanh \left(d_{0}\right)}\left(\overline{\mathrm{D}}_{x_{1}}(v) \wedge \overline{\overline{\mathrm{D}}_{x_{1}}(v)}-\mathrm{D}_{x_{1}}(v) \wedge \overline{\mathrm{D}_{x_{1}}(v)}\right) \\
-\frac{1}{16 \tanh ^{2}\left(d_{0}\right)}\left|\mathbf{u}\left[q_{0}\right] \wedge \overline{\overline{\mathrm{D}}_{x_{1}}(v)}\right|^{2}
\end{gathered}
$$

for $v \in T_{x_{1}} \mathcal{T}_{g, m}$. From (40) and (46), (54) and (56),

$$
\begin{aligned}
& \mathcal{L}\left(u_{x_{0}}\right)\left[\boldsymbol{\nu}\left(x_{1}\right), \overline{\boldsymbol{\nu}\left(x_{1}\right)}\right] \\
& =\frac{\sqrt{-1}}{4 \tanh \left(d_{0}\right)} \frac{\mathbf{u}\left[q_{0}\right]}{\sinh \left(2 d_{0}\right)} \wedge \frac{\overline{\mathbf{u}\left[q_{0}\right]}}{\sinh \left(2 d_{0}\right)}-\frac{1}{16 \tanh ^{2}\left(d_{0}\right)}\left|\mathbf{u}\left[q_{0}\right] \wedge \frac{\overline{\mathbf{u}\left[q_{0}\right]}}{\sinh \left(2 d_{0}\right)}\right|^{2}=0 .
\end{aligned}
$$

From Lemma 7.2, the Levi form of $u_{x_{0}}$ is non-negative on $H_{x_{1}}^{1,0}$. Applying the calculation in Proposition 7.1 to (57), we also deduce that the normal vector $\boldsymbol{\nu}\left(x_{1}\right)$ 
is perpendicular to $H_{x_{1}}^{1,0}$ with respect to the Levi form of $u_{x_{0}}$. Therefore, the Levi form of $u_{x_{0}}$ is non-negative on the whole $T_{x_{1}} \mathcal{T}_{g, m}$.

7.7. Proof of Corollary 1.1. As mentioned in the introduction, we identify $\mathcal{T}_{g, m}$ with the Bers slice with base point $x_{0} \in \mathcal{T}_{g, m}$. Then, $\mathcal{T}_{g, m}$ is a hyperconvex domain. Notice that for $x, y \in \mathcal{T}_{g, m}$,

$$
\frac{g_{\mathcal{T}_{g, m}}(x, z)}{g_{\mathcal{T}_{g, m}}(y, z)}=e^{-2\left(d_{T}(x, z)-d_{T}(y, z)\right)}(1+o(1)) \leq e^{2 d_{T}(x, y)}(1+o(1))
$$

when $z \rightarrow \partial \mathcal{T}_{g, m}$. Therefore, $\boldsymbol{\delta}_{\mathcal{T}_{g, m}}(x, y) \leq 2 d_{T}(x, y)$.

On the other hand, when we consider a divergent sequence $\left\{x_{n}\right\}_{n}$ in $\mathcal{T}_{g, m}$ along the Teichmüller geodesic connecting $x$ and $y$ with $d_{T}\left(x, x_{n}\right)<d_{T}\left(y, x_{n}\right)$, we have

$$
\frac{g_{\mathcal{T}_{g, m}}\left(x, x_{n}\right)}{g_{\mathcal{T}_{g, m}}\left(y, x_{n}\right)}=e^{-2\left(d_{T}\left(x, x_{n}\right)-d_{T}\left(y, x_{n}\right)\right)}(1+o(1))=e^{2 d_{T}(x, y)}(1+o(1))
$$

as $n \rightarrow \infty$, and hence $2 d_{T}(x, y) \leq \boldsymbol{\delta}_{\mathcal{T}_{g, m}}(x, y)$.

\section{TOPOLOGICAL DESCRIPTION OF THE LEVI FORM}

The space $\mathcal{M F}$ carries a natural symplectic structure with the Thurston symplectic form $\omega_{T h}$ (cf. [50]). Dumas [14, Theorem 5.3] introduced a Kähler (symplectic) structure on each stratum of $\mathcal{Q}_{x_{0}}$ discussed in 6 which defined the Levi form of the $L^{1}$-norm on $\mathcal{Q}_{x_{0}}$, and it was observed that the Hubbard-Masur homeomorphism (17) is a real-analytic symplectomorphism on each stratum of $\mathcal{Q}_{x_{0}}$ (cf. [14, Theorem 5.8]). In fact, when $q_{0} \in \mathcal{Q}_{x_{0}}$ is generic, Dumas showed that the Hubbard-Masur homeomorphism (7) is a diffeomorphism around $q_{0}$ and satisfies

$$
\begin{aligned}
\omega_{T h}\left(d \mathcal{V}_{x_{0}}\left(\psi_{1}\right), d \mathcal{V}_{x_{0}}\left(\psi_{2}\right)\right) & =\operatorname{Im} \int_{M_{0}} \frac{\psi_{1} \overline{\psi_{2}}}{4\left|q_{0}\right|} \\
& =\frac{1}{8} \int_{\tilde{M}_{q_{0}}} \operatorname{Re}\left(\frac{\pi_{q_{0}}^{*}\left(\psi_{1}\right)}{\omega_{q_{0}}}\right) \wedge \operatorname{Re}\left(\frac{\pi_{q_{0}}^{*}\left(\psi_{2}\right)}{\omega_{q_{0}}}\right)
\end{aligned}
$$

for $\psi_{1}, \psi_{2} \in T_{q_{0}} \mathcal{Q}_{x_{0}}=\mathcal{Q}_{x_{0}}$ (cf. (12) and [14, §5.2]).

Remark 8.1. Dumas [14] discussed the Hubbard-Masur homeomorphism (7) by assigning the horizontal foliations to quadratic differentials in accordance with Hubbard and Masur's original discussion. The original Hubbard-Masur homeomorphism $\mathcal{H}_{x_{0}}: \mathcal{Q}_{x_{0}} \rightarrow \mathcal{M F}$ satisfies $\mathcal{V}_{x_{0}}(q)=\mathcal{H}_{x_{0}}(-q)$. Hence, $d \mathcal{V}_{x_{0}}(\psi)=-d \mathcal{H}_{x_{0}}(\psi)$ for $\psi \in \mathcal{Q}_{x_{0}}=T_{q_{0}} \mathcal{Q}_{x_{0}}$. Thus, the formula (58) also holds in our case.

Let us go back to the notion in $\$ 7.2$. Notice that

$$
\begin{aligned}
\mathbf{v}_{2} \wedge \overline{\mathbf{v}_{2}}-\mathbf{v}_{1} \wedge \overline{\mathbf{v}_{1}} & =\left(\mathbf{v}_{2}+\overline{\mathbf{v}}_{1}\right) \wedge\left(\overline{\mathbf{v}}_{2}+\mathbf{v}_{1}\right)=\left(\operatorname{Re}\left(\mathbf{u}\left[q_{\lambda}\right]\right)\right)_{\bar{\lambda}} \wedge \overline{\left(\operatorname{Re}\left(\mathbf{u}\left[q_{\lambda}\right]\right)\right)_{\bar{\lambda}}} \\
& =-\frac{\sqrt{-1}}{2} \operatorname{Re}\left(\mathbf{u}\left[q_{\lambda}\right]_{\xi_{1}}\right) \wedge \operatorname{Re}\left(\mathbf{u}\left[q_{\lambda}\right]_{\xi_{2}}\right)
\end{aligned}
$$

at $\lambda=0$, where $\lambda=\xi_{1}+\xi_{2} \sqrt{-1}$. Therefore, when

$$
q_{\lambda}=\Xi_{x_{0}}(g(\lambda))=q_{0}+\xi_{1} \psi_{1}+\xi_{2} \psi_{2}+o(|\lambda|)
$$

as $\lambda=\xi_{1}+\xi_{2} \sqrt{-1} \rightarrow 0$, from (40), (46), (58), and (59), we deduce the topological description of the Levi form of the pluricomplex Green function $u_{x_{0}}$ on $\mathcal{T}_{g, m}$ :

$$
\mathcal{L}\left(u_{x_{0}}\right)[v, \bar{v}]=\frac{1}{\left\|q_{0}\right\|} \omega_{T h}\left(d \mathcal{V}_{x_{0}}\left(\psi_{1}\right), d \mathcal{V}_{x_{0}}\left(\psi_{2}\right)\right)-\frac{\left|\mathbf{u}\left[q_{0}\right] \wedge \overline{\overline{\mathrm{D}}_{x_{1}}(v)}\right|^{2}}{16\left\|q_{0}\right\|^{2}},
$$


where

$$
\begin{aligned}
\mathbf{u}\left[q_{0}\right] \wedge \overline{\overline{\mathrm{D}}_{x_{1}}(v)}=8\left(\omega_{T h}\left(d \mathcal{V}_{x_{0}}\left(q_{0}\right), d \mathcal{V}_{x_{0}}\left(\psi_{2}-\sqrt{-1} \psi_{1}\right)\right)\right. \\
\left.+\sqrt{-1} \omega_{T h}\left(d \mathcal{V}_{x_{0}}\left(q_{0}\right), d \mathcal{V}_{x_{0}}\left(\psi_{1}+\sqrt{-1} \psi_{2}\right)\right)\right) .
\end{aligned}
$$

In particular, when $v \in H_{x_{1}}^{1,0}$ for $x_{1}=g(0)$, we conclude

$$
\mathcal{L}\left(u_{x_{0}}\right)[v, \bar{v}]=\frac{1}{\left\|q_{0}\right\|} \omega_{T h}\left(d \mathcal{V}_{x_{0}}\left(\psi_{1}\right), d \mathcal{V}_{x_{0}}\left(\psi_{2}\right)\right)
$$

As a corollary, we deduce

$$
\omega_{T h}\left(d \mathcal{V}_{x_{0}}\left(\psi_{1}\right), d \mathcal{V}_{x_{0}}\left(\psi_{2}\right)\right) \geq \frac{\left|\mathbf{u}\left[q_{0}\right] \wedge \overline{\bar{D}}_{x_{1}}(v)\right|^{2}}{16\left\|q_{0}\right\|} \geq 0 .
$$

From the definition, $d \mathcal{V}_{x_{0}}\left(\psi_{i}\right) \in T_{v\left(q_{0}\right)} \mathcal{M F}$ is the infinitesimal transverse cocycle (in the sense of Bonahon [5]) of the initial differentials $\left\{q_{\lambda}\right\}_{\lambda}$ associated along the $\xi_{i^{-}}$ direction at $\lambda=0$. Thus, the non-negativity derived from (61) of the Thurston symplectic pairing between the infinitesimal transverse cocycles $d \mathcal{V}_{x_{0}}\left(\psi_{1}\right)$ and $d \mathcal{V}_{x_{0}}\left(\psi_{2}\right)$ is a necessary condition to describe complex analytic deformations of Teichmüller mappings from the topological aspect in Teichmüller theory.

\section{Appendix}

In this section, we shall check Proposition 6.2. As noted before, Proposition 6.2 is proved under the additional assumption that $u$ is of class $C^{2}$ by Blanchet 3 . Chirka [8] extends Blanchet's result as follows: When $\Omega$ and $V$ are taken as in Proposition 6.2 a subharmonic function on $\Omega$ which is plurisubharmonic on $\Omega \backslash V$ is plurisubharmonic on $\Omega$ without any smoothness assumption. Hence, Proposition 6.2 follows from the following proposition which appears to be well known. However, the author cannot find any suitable reference and gives a proof for completeness.

Proposition 9.1. Let $\Omega$ be a domain in $\mathbb{R}^{N}$ and let $V$ be a $C^{1}$-submanifold of $\Omega$ with positive codimension. When a $C^{1}$-function $u$ on $\Omega$ is subharmonic on $\Omega \backslash V$, $u$ is subharmonic on $\Omega$.

Proof. Since the subharmonicity is a local condition, we may assume that $\Omega$ is a ball centered at $z_{0}$ and $V$ is a hypersurface ([30, Theorem 2.4.1]). Let $z_{0} \in V$ and let $\mathbf{n}$ be the normal vector to $V$ at $z_{0}$. By applying the inverse mapping theorem, we may also assume that

- for small $\delta_{0}>0, V_{t}=V+t \mathbf{n}\left(|t|<\delta_{0}\right)$ separates $\Omega$ into two domains, and intersects $\partial \Omega$ transversely;

- $V_{t} \cap V_{t^{\prime}}=\emptyset\left(t \neq t^{\prime}\right)$; and

- for any $0<\delta<\delta_{0}$, the union $N(\delta)=\cup_{|t|<\delta} V_{t}$ forms an open set.

For a domain $E$ and a constant $r>0$ we set $E_{r}=\{x \in E \mid \operatorname{dist}(x, \partial E)>r\}$. Let $\Omega_{\delta} \backslash N(\delta)=\Omega_{\delta}^{+} \sqcup \Omega_{\delta}^{-}$and $F_{\delta}^{ \pm}=\overline{\Omega_{\delta}^{ \pm}} \cap V_{ \pm \delta}$ (we assume $F_{\delta}^{ \pm} \neq \emptyset$ ). Then, $\partial \Omega_{\delta}^{ \pm}=\left(\partial \Omega_{\delta} \cap \overline{\Omega_{\delta}^{ \pm}}\right) \cup F_{\delta}^{ \pm}$.

Let $\epsilon>0$. Let $u_{\epsilon}=\chi_{\epsilon} * u$ be the convolution where $\chi_{\epsilon}$ is a standard smoothing kernel (mollifier) supported on the ball centered at the origin with radius $\epsilon$. Then, $u_{\epsilon}$ is a smooth function defined on $\Omega_{\epsilon}$, and subharmonic on $(\Omega \backslash V)_{\epsilon}$. Furthermore, since $u$ is of class $C^{1}, u_{\epsilon}$ and its partial derivative $\left(u_{\epsilon}\right)_{x_{i}}=\chi_{\epsilon} * u_{x_{i}}$ converge to $u$ and $u_{x_{i}}$ uniformly on any compact subset of $\Omega$, respectively (cf. [30, $\left.\S 2.5\right]$ ). 
Let $\varphi \in C_{0}^{\infty}(\Omega)$ with $\varphi \geq 0$. Fix $\delta>0$ with $\delta<\delta_{0}$ such that $\operatorname{supp}(\varphi) \subset \Omega_{\delta}$ for $s= \pm$. When $\delta$ is sufficiently small, $V_{t}(|t| \leq \delta)$ intersects $\partial \Omega_{\delta}$ transversely. If $\epsilon>0$ is sufficiently small, $\overline{\Omega_{\delta}^{s}} \subset(\Omega \backslash V)_{\epsilon}$ for $s= \pm$ because $\Omega_{\delta}^{s}$ is relatively compact in $\Omega \backslash V$. Since $\varphi \equiv 0$ around $\partial \Omega_{\delta}$, by Green's Theorem,

$$
0 \leq \int_{\Omega_{\delta}^{s}} \varphi \Delta u_{\epsilon} d V=\int_{F_{\delta}^{s}}\left(\varphi\left(D_{n} u_{\epsilon}\right)-u_{\epsilon}\left(D_{n} \varphi\right)\right) d S+\int_{\Omega_{\delta}^{s}} u_{\epsilon} \Delta \varphi d V
$$

for $s= \pm$, where $d V$ is the Lebesgue measure on $\mathbb{R}^{N}, d S$ is the surface area element on $F_{\delta}^{s}$, and $D_{n}$ is the outer unit normal derivative on $F_{\delta}^{s}$ with respect to $\Omega_{\delta}^{s}$ (cf. [23, §1.2] and [37, Theorem 16.25]). By letting $\epsilon \rightarrow 0$, we obtain

$$
0 \leq \sum_{s= \pm} \int_{F_{\delta}^{s}}\left(\varphi\left(D_{n} u\right)-u\left(D_{n} \varphi\right)\right) d S+\sum_{s= \pm} \int_{\Omega_{\delta}^{s}} u \Delta \varphi d V .
$$

Since $V$ separates $\Omega_{\delta}^{+}$and $\Omega_{\delta}^{-}$and $u$ and $\varphi$ are of class $C^{1}$ on $\Omega$,

$$
\lim _{\delta \rightarrow 0} \int_{F_{\delta}^{+}}\left(\varphi\left(D_{n} u\right)-u\left(D_{n} \varphi\right)\right) d S=-\lim _{\delta \rightarrow 0} \int_{F_{\delta}^{-}}\left(\varphi\left(D_{n} u\right)-u\left(D_{n} \varphi\right)\right) d S .
$$

Therefore, we conclude that

$$
0 \leq \int_{\Omega} u \Delta \varphi d V
$$

by letting $\delta \rightarrow 0$. This means that $\Delta u \geq 0$ on $\Omega$ in the sense of distribution, and $u$ is subharmonic on $\Omega$ ([30, Theorem 2.5.8]).

\section{ACKNOWLEDGMENTS}

The author thanks Professor Ken'ichi Ohshika for fruitful discussions. The author also thanks Professor Masanori Adachi for directing him to Blanchet's and Chirka's papers [3] and [8], and Professor Hiroshi Yamaguchi for his warm advice and encouragement.

\section{REFERENCES}

[1] William Abikoff, The real analytic theory of Teichmüller space, Lecture Notes in Mathematics, vol. 820, Springer, Berlin, 1980. MR590044

[2] Lipman Bers, Correction to "Spaces of Riemann surfaces as bounded domains", Bull. Amer. Math. Soc. 67 (1961), 465-466, DOI 10.1090/S0002-9904-1961-10637-X. MR130972

[3] Pierre Blanchet, On removable singularities of subharmonic and plurisubharmonic functions, Complex Variables Theory Appl. 26 (1995), no. 4, 311-322, DOI 10.1080/17476939508814792. MR.1315864

[4] Albert Boggess, CR manifolds and the tangential Cauchy-Riemann complex, Studies in Advanced Mathematics, CRC Press, Boca Raton, FL, 1991. MR.1211412

[5] Francis Bonahon, Shearing hyperbolic surfaces, bending pleated surfaces and Thurston's symplectic form (English, with English and French summaries), Ann. Fac. Sci. Toulouse Math. (6) 5 (1996), no. 2, 233-297. MR 1413855

[6] Jeffrey F. Brock, Richard D. Canary, and Yair N. Minsky, The classification of Kleinian surface groups, II: The ending lamination conjecture, Ann. of Math. (2) 176 (2012), no. 1, 1-149, DOI 10.4007/annals.2012.176.1.1. MR.2925381

[7] Richard D. Canary, Introductory bumponomics: the topology of deformation spaces of hyperbolic 3-manifolds, Teichmüller theory and moduli problem, Ramanujan Math. Soc. Lect. Notes Ser., vol. 10, Ramanujan Math. Soc., Mysore, 2010, pp. 131-150. MR2667553

[8] E. M. Chirka, On the removal of subharmonic singularities of plurisubharmonic functions, Proceedings of Conference on Complex Analysis (Bielsko-Biała, 2001), Ann. Polon. Math. 80 (2003), 113-116, DOI 10.4064/ap80-0-8. MR.1972852 
[9] Dan Coman, Boundary behavior of the pluricomplex Green function, Ark. Mat. 36 (1998), no. 2, 341-353, DOI 10.1007/BF02384773. MR1650450

[10] Jean-Pierre Demailly, Mesures de Monge-Ampère et mesures pluriharmoniques (French), Math. Z. 194 (1987), no. 4, 519-564, DOI 10.1007/BF01161920. MR881709

[11] K. Diederich and G. Herbort, Quantitative estimates for the Green function and an application to the Bergman metric (English, with English and French summaries), Ann. Inst. Fourier (Grenoble) $\mathbf{5 0}$ (2000), no. 4, 1205-1228. MR.1799743

[12] Travaux de Thurston sur les surfaces (French), Astérisque, vol. 66, Société Mathématique de France, Paris, 1979. Séminaire Orsay; With an English summary. MR568308

[13] A. Douady and J. Hubbard, On the density of Strebel differentials, Invent. Math. 30 (1975), no. 2, 175-179, DOI 10.1007/BF01425507. MR396936

[14] David Dumas, Skinning maps are finite-to-one, Acta Math. 215 (2015), no. 1, 55-126, DOI 10.1007/s11511-015-0129-6. MR.3413977

[15] Clifford J. Earle, The Teichmüller distance is differentiable, Duke Math. J. 44 (1977), no. 2, 389-397. MR 445013

[16] H. M. Farkas and I. Kra, Riemann surfaces, 2nd ed., Graduate Texts in Mathematics, vol. 71, Springer-Verlag, New York, 1992. MR1139765

[17] Frederick P. Gardiner, Teichmüller theory and quadratic differentials, Pure and Applied Mathematics (New York), John Wiley \& Sons, Inc., New York, 1987. A Wiley-Interscience Publication. MR903027

[18] Frederick P. Gardiner and Howard Masur, Extremal length geometry of Teichmüller space, Complex Variables Theory Appl. 16 (1991), no. 2-3, 209-237, DOI 10.1080/17476939108814480. MR.1099913

[19] Roger Godement, Topologie algébrique et théorie des faisceaux (French), Actualit'es Sci. Ind. No. 1252. Publ. Math. Univ. Strasbourg. No. 13, Hermann, Paris, 1958. MR0102797

[20] Phillip Griffiths and Joseph Harris, Principles of algebraic geometry, Wiley-Interscience [John Wiley \& Sons], New York, 1978. Pure and Applied Mathematics. MR507725

[21] Allen Hatcher, Algebraic topology, Cambridge University Press, Cambridge, 2002. MR.1867354

[22] Gregor Herbort, The pluricomplex Green function on some regular pseudoconvex domains, Ann. Polon. Math. 110 (2014), no. 3, 209-226, DOI 10.4064/ap110-3-1. MR3172935

[23] Lester L. Helms, Potential theory, Universitext, Springer-Verlag London, Ltd., London, 2009. MR.2526019

[24] John Hubbard and Howard Masur, Quadratic differentials and foliations, Acta Math. 142 (1979), no. 3-4, 221-274, DOI 10.1007/BF02395062. MR523212

[25] John Hamal Hubbard, Teichmüller theory and applications to geometry, topology, and dynamics. Vol. 1, Matrix Editions, Ithaca, NY, 2006. Teichmüller theory; With contributions by Adrien Douady, William Dunbar, Roland Roeder, Sylvain Bonnot, David Brown, Allen Hatcher, Chris Hruska and Sudeb Mitra; With forewords by William Thurston and Clifford Earle. MR 2245223

[26] Y. Imayoshi and M. Taniguchi, An introduction to Teichmüller spaces, Springer-Verlag, Tokyo, 1992. Translated and revised from the Japanese by the authors. MR.1215481

[27] Yujiro Kawamata, On deformations of compactifiable complex manifolds, Math. Ann. 235 (1978), no. 3, 247-265, DOI 10.1007/BF01420124. MR499279

[28] Steven P. Kerckhoff, The asymptotic geometry of Teichmüller space, Topology 19 (1980), no. 1, 23-41, DOI 10.1016/0040-9383(80)90029-4. MR559474

[29] M. Klimek, Extremal plurisubharmonic functions and invariant pseudodistances (English, with French summary), Bull. Soc. Math. France 113 (1985), no. 2, 231-240. MR820321

[30] Maciej Klimek, Pluripotential theory, London Mathematical Society Monographs. New Series, vol. 6, The Clarendon Press, Oxford University Press, New York, 1991. Oxford Science Publications. MR.1150978

[31] Shoshichi Kobayashi and Katsumi Nomizu, Foundations of differential geometry. Vol. II, Wiley Classics Library, John Wiley \& Sons, Inc., New York, 1996. Reprint of the 1969 original; A Wiley-Interscience Publication. MR1393941

[32] Kunihiko Kodaira, Complex manifolds and deformation of complex structures, Grundlehren der Mathematischen Wissenschaften [Fundamental Principles of Mathematical Sciences], vol. 283, Springer-Verlag, New York, 1986. Translated from the Japanese by Kazuo Akao; With an appendix by Daisuke Fujiwara. MR 815922 
[33] Yohei Komori and Jouni Parkkonen, On the shape of Bers-Maskit slices, Ann. Acad. Sci. Fenn. Math. 32 (2007), no. 1, 179-198. MR2297885

[34] Irwin Kra, The Carathéodory metric on abelian Teichmüller disks, J. Analyse Math. 40 (1981), 129-143 (1982), DOI 10.1007/BF02790158. MR659787

[35] S. L. Krushkal', Strengthening pseudoconvexity of finite-dimensional Teichmüller spaces, Math. Ann. 290 (1991), no. 4, 681-687, DOI 10.1007/BF01459267. MR.1119946

[36] Samuel L. Krushkal, The Green function of Teichmüller spaces with applications, Bull. Amer. Math. Soc. (N.S.) 27 (1992), no. 1, 143-147, DOI 10.1090/S0273-0979-1992-00294X. MR 1142683

[37] John M. Lee, Introduction to smooth manifolds, 2nd ed., Graduate Texts in Mathematics, vol. 218, Springer, New York, 2013. MR2954043

[38] Howard Masur, Interval exchange transformations and measured foliations, Ann. of Math. (2) 115 (1982), no. 1, 169-200, DOI 10.2307/1971341. MR644018

[39] Howard Masur, Random walks on Teichmuller space and the mapping class group, J. Anal. Math. 67 (1995), 117-164, DOI 10.1007/BF02787787. MR.1383491

[40] Howard Masur, The Teichmüller flow is Hamiltonian, Proc. Amer. Math. Soc. 123 (1995), no. 12, 3739-3747, DOI 10.2307/2161902. MR.1283559

[41] Howard Masur and John Smillie, Hausdorff dimension of sets of nonergodic measured foliations, Ann. of Math. (2) 134 (1991), no. 3, 455-543, DOI 10.2307/2944356. MR1135877

[42] Yair N. Minsky, The classification of punctured-torus groups, Ann. of Math. (2) 149 (1999), no. 2, 559-626, DOI 10.2307/120976. MR1689341

[43] Yair Minsky, The classification of Kleinian surface groups. I. Models and bounds, Ann. of Math. (2) 171 (2010), no. 1, 1-107, DOI 10.4007/annals.2010.171.1. MR2630036

[44] Hideki Miyachi, Cusps in complex boundaries of one-dimensional Teichmüller space, Conform. Geom. Dyn. 7 (2003), 103-151, DOI 10.1090/S1088-4173-03-00065-1. MR2023050

[45] Hideki Miyachi, Unification of extremal length geometry on Teichmüller space via intersection number, Math. Z. 278 (2014), no. 3-4, 1065-1095, DOI 10.1007/s00209-014-1346-y. MR.3278905

[46] Hideki Miyachi, Extremal length functions are log-plurisubharmonic, In the tradition of Ahlfors-Bers. VII, Contemp. Math., vol. 696, Amer. Math. Soc., Providence, RI, 2017, pp. 225-250, DOI 10.1090/conm/696/14024. MR.3715450

[47] Hideki Miyachi. Teichmüller theory, Thurston theory, Extremal length geometry and Complex analysis. submitted (2018).

[48] Hideki Miyachi, Pluripotential theory on Teichmüller space II - Poisson integral formula, eprint arXiv:1810.04343 (2018).

[49] Subhashis Nag, The complex analytic theory of Teichmüller spaces, Canadian Mathematical Society Series of Monographs and Advanced Texts, John Wiley \& Sons, Inc., New York, 1988. A Wiley-Interscience Publication. MR927291

[50] R. C. Penner and J. L. Harer, Combinatorics of train tracks, Annals of Mathematics Studies, vol. 125, Princeton University Press, Princeton, NJ, 1992. MR1144770

[51] E. A. Poletskiı̌ and B. V. Shabat, Invariant metrics (Russian), Current problems in mathematics. Fundamental directions, Vol. 9 (Russian), Itogi Nauki i Tekhniki, Akad. Nauk SSSR, Vsesoyuz. Inst. Nauchn. i Tekhn. Inform., Moscow, 1986, pp. 73-125, 292. MR860610

[52] Mary Rees, Teichmüller distance for analytically finite surfaces is $C^{2}$, Proc. London Math. Soc. (3) 85 (2002), no. 3, 686-716, DOI 10.1112/S0024611502013746. MR1936817

[53] H. L. Royden, Automorphisms and isometries of Teichmüller space, Advances in the Theory of Riemann Surfaces (Proc. Conf., Stony Brook, N.Y., 1969), Ann. of Math. Studies, No. 66. Princeton Univ. Press, Princeton, N.J., 1971, pp. 369-383. MR0288254

[54] M.-H. Schwartz, Lectures on stratification of complex analytic sets, Tata Institute of Fundamental Research Lectures on Mathematics, No. 38, Tata Institute of Fundamental Research, Bombay, 1966. MR0214811

[55] Hiroshige Shiga, On analytic and geometric properties of Teichmüller spaces, J. Math. Kyoto Univ. 24 (1984), no. 3, 441-452, DOI 10.1215/kjm/1250521274. MR766636

[56] Jean-Luc Stehlé, Fonctions plurisousharmoniques et convexité holomorphe de certains fibrés analytiques (French), C. R. Acad. Sci. Paris Sér. A 279 (1974), 235-238. MR0374493

[57] Kurt Strebel, Quadratic differentials, Ergebnisse der Mathematik und ihrer Grenzgebiete (3) [Results in Mathematics and Related Areas (3)], vol. 5, Springer-Verlag, Berlin, 1984. MR743423 
[58] William A. Veech, The Teichmüller geodesic flow, Ann. of Math. (2) 124 (1986), no. 3, 441-530, DOI 10.2307/2007091. MR866707

[59] William A. Veech, Moduli spaces of quadratic differentials, J. Analyse Math. 55 (1990), 117171, DOI 10.1007/BF02789200. MR1094714

[60] Hassler Whitney, Tangents to an analytic variety, Ann. of Math. (2) 81 (1965), 496-549, DOI 10.2307/1970400. MR192520

Division of Mathematical and Physical Sciences, Graduate School of Natural Science \& Technology, Kanazawa University, Kakuma-Machi, KanazaWa, IshikaWa, 920-1192, JAPAN

Email address: miyachi@se.kanazawa-u.ac.jp 